\title{
La Blancharderie àArgentré-du-Plessis (35) : un site de fond de vallée à la fin de l'âge du Fer sur le tracé de la LGV Rennes-Le Mans
}

La Blancharderie - Argentré-du-Plessis (35): a valley bottom site of Late Iron Age on the new high-speed railway between Rennes and Le Mans

El Blancharderie en Argentré-du-Plessis (35): un sitio de fondo de valle al final de la Edad del Hierro en el trazado del tren de alta velocidad Rennes-Le Mans La Blancharderie bei Argentré-du-Plessis (Ille-et-Vilaine, Frankreich): ein späteisenzeitlicher Fundplatz in einem Tälchen im Bereich der Schnellzugtrasse zwischen Rennes und Le Mans

\section{Sébastien Toron et Océane Lierville}

\section{OpenEdition} Journals

Édition électronique

URL : http://journals.openedition.org/rao/3051

DOI : 10.4000/rao.3051

ISBN : 978-2-7535-5014-8

ISSN : $1775-3732$

Éditeur

Presses universitaires de Rennes

Édition imprimée

Date de publication : 31 décembre 2015

Pagination : 261-284

ISBN : 978-2-7535-5012-4

ISSN : 0767-709X

\section{Référence électronique}

Sébastien Toron et Océane Lierville, «La Blancharderie àArgentré-du-Plessis (35) : un site de fond de vallée à la fin de l'âge du Fer sur le tracé de la LGV Rennes-Le Mans ", Revue archéologique de l'Ouest [En ligne], 32 | 2015, mis en ligne le 28 avril 2018, consulté le 02 mars 2021. URL : http:// journals.openedition.org/rao/3051 ; DOI : https://doi.org/10.4000/rao.3051 


\title{
La Blancharderie à Argentré-du-Plessis (Ille-et-Vilaine) : un site de fond de vallée à la fin de l'âge du Fer sur le tracé de la LGV Rennes-Le Mans
}

\author{
La Blancharderie - Argentré-du-Plessis (Ille-et-Vilaine): \\ A Valley Bottom Site of Late Iron Age \\ on the New High-speed Railway between Rennes and Le Mans
}

\author{
Sébastien Toron ${ }^{a}$ et Océane Lierville ${ }^{a}$
}

\begin{abstract}
Résumé : La fouille archéologique d'Argentré-du-Plessis a été engagée dans le cadre de l’aménagement de la ligne LGV Rennes-Le Mans. Parmi les sites mis au jour sur ce tracé linéaire, celui de La Blancharderie offre la particularité de se situer en fond de vallée à proximité immédiate de la confluence de deux ruisseaux encore en activité. En bordure de thalweg, dans une zone humide qui connut plusieurs transformations, a pu être mis au jour la limite d'extension d'un établissement gaulois qui évolue en continu au moins du début du III ${ }^{e}$ siècle jusqu'au début du I ${ }^{\text {er }}$ siècle avant notre ère. L'occupation se caractérise par la mise en place d'un système de fossés rectilignes délimitant un vaste enclos trapézoïdal. Son extrémité a été dégagée dans l'emprise de la fouille sur une surface de $850 \mathrm{~m}^{2}$, tandis qu'il se poursuit sans nul doute vers le plateau au sud-ouest. L'intérêt de cet enclos, outre sa situation en bordure d'une zone humide, réside dans la multiplication des constructions qui révèle une succession spatiale et temporelle d'habitations dans un espace réduit. Diverses architectures sur tranchées de fondations, parois à poteaux jointifs et bâtiments sur poteaux plantés ont pu être dégagés.
\end{abstract}

\begin{abstract}
The archaeological excavation of Argentré-du-Plessis was undertaken in the context of the new high-speed railway between Rennes and Le Mans. Among the sites investigated along this linear corridor, La Blancharderie excavation has the particularity of being situated in the valley floor close to the confluence of two still active streams. The site is located on the edge of a thalweg within an ancient wetland that underwent several transformations from the third to the beginning of the first century $B C$. The main occupation is characterized by the implantation of a rectilinear ditches system delimiting trapezoid-shaped enclosure. Covering an area of $850 \mathrm{~m}^{2}$ within the excavation area, the enclosure continues to southwest outside of the current area of investigation. The major interest of the enclosure resides in the multitude of construction episodes observed, revealing a spatial and temporal succession of buildings in a limited space. Several buildings types were identified, including foundation trenches, post walls, and pile construction techniques.
\end{abstract}

Mots clés : Bretagne, zone humide, fond de vallée, enclos, habitat, tranchée de fondation, La Tène finale, céramique, plaque foyère, meule.

Keyword: Brittany, wetland, bottom valley, enclosure, settlement, foundation trench, Late Iron Age, ceramic, hearth slab, quern.

\section{INTRODUCTION}

\section{Contexte de découverte}

Localisée dans le département de l'Ille-et-Vilaine, entre Rennes et Laval, la commune d'Argentré-du-Plessis se trouve en bordure de la Mayenne (fig. 1). Le site de $L a$ Blancharderie, situé à trois kilomètres au sud de la commune, a été découvert à l'occasion des opérations de diagnostics archéologiques engagées dès 2010 par l'Inrap sur les deux cent quatorze kilomètres de linéaire que représente l'aménagement de la Ligne à Grande Vitesse Le Mans-Rennes.

a Éveha Ouest Atlantique - Études et valorisations archéologiques, 23, rue des Maréchales, 35132 Vezin-le-Coquet-Chercheurs associés à l'UMR 6566 CreAAH, université de Rennes 1. (sebastien.toron@eveha.fr) (oceane.lierville@eveha.fr) 

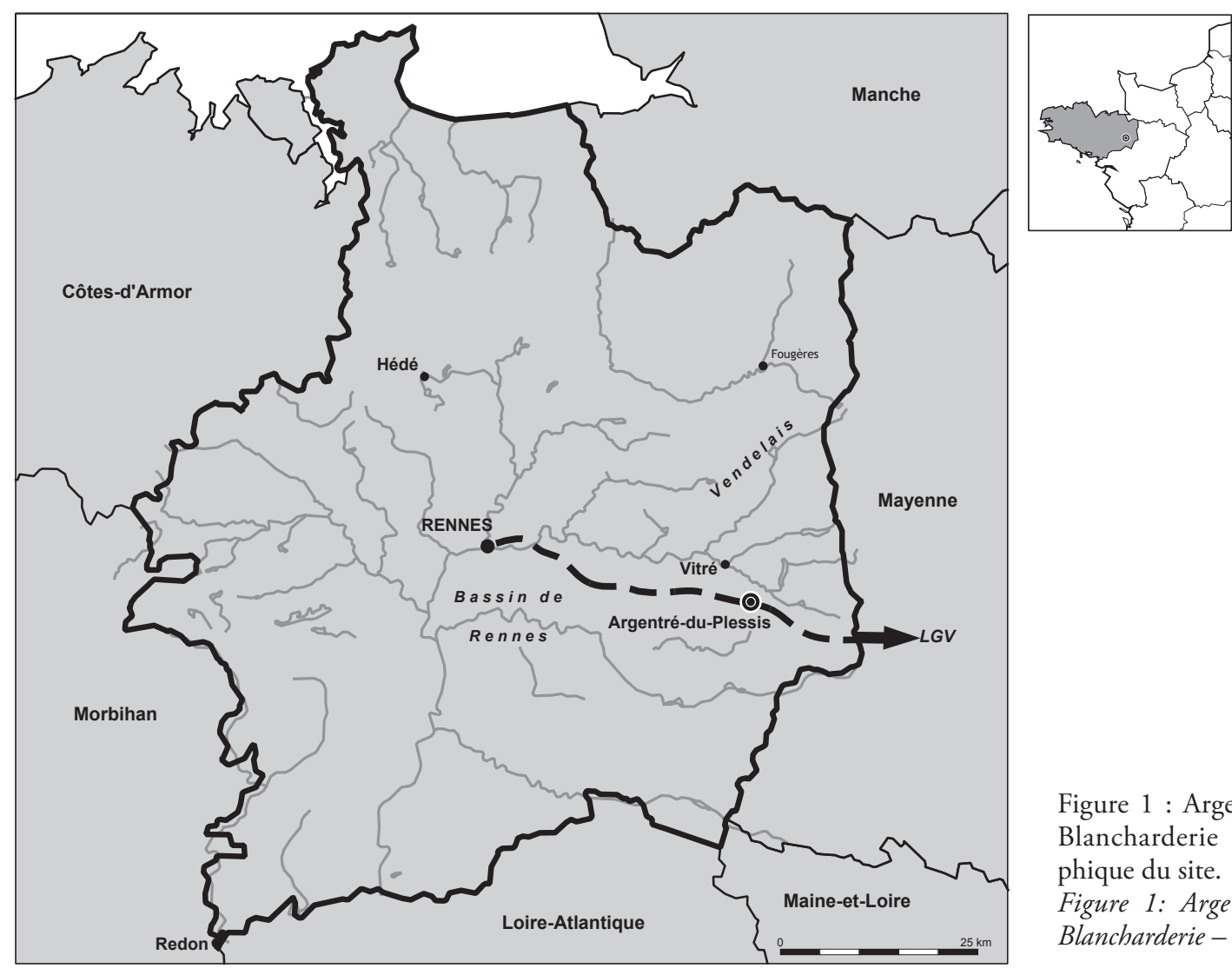

Figure 1 : Argentré-du-Plessis (35), La Blancharderie - Localisation géographique du site.

Figure 1: Argentré-du-Plessis (35), La Blancharderie - Site location.

Ce tracé, découpé en dix-neuf sections, a livré de nombreux sites archéologiques notamment pour les périodes protohistoriques. Argentré-du-Plessis se situe sur la quatrième section de ce découpage; le site de La Blancharderie est le cinquième et dernier mis au jour sur cette section.

La particularité de La Blancharderie est sa localisation dans un environnement d'étangs et de ruisseaux encore en activité. Sa situation en fond de vallée est marquée aujourd'hui par une altitude constante de $77 \mathrm{~m} \mathrm{NGF}$ du nord au sud. Le site est en position encaissée dans un delta où le micro-relief culmine en moyenne à $85 \mathrm{~m}$ NGF. C'est dans ce contexte environnemental particulier qu'a été mis au jour un établissement rural du second âge du Fer fouillé entre mai et juillet 2011 sur une superficie d'un hectare environ.

\section{La trame des sites ruraux de la Tène au sud-est du bassin de Rennes}

Sur les quatre sections du projet d'aménagement en Ille-etVilaine, ce ne sont pas moins de onze sites de l'âge du Fer qui ont été mis en évidence lors des diagnostics de l'Inrap (fig. 2). Sur la première section partant de Rennes a été diagnostiqué le site de La Salmondière à Cesson-Sévigné (Barbeau, 2011b : 55-68) qui a fait l'objet d'une fouille par J.-C. Durand en 2012. Il contenait notamment un vaste domaine rural com- posé de plusieurs enclos laténiens. À La Rodais, commune de Noyal-sur-Vilaine, le diagnostic a révélé les traces d'un bâtiment et peut-être d'un enclos de la fin de La Tène (Barbeau, 2011b : 27-32).

Sur la deuxième section se trouve l'établissement rural de Domagné La Lande Barbot fouillé par Éveha en 2012. Ce site, occupé dès la fin du premier âge du Fer, se structure autour d'un système d'enclos durant la deuxième moitié du III $^{\mathrm{e}}$ siècle et est restructuré au I ${ }^{\mathrm{er}}$ siècle av. J.-C. (Toron, 2014). À une dizaine de kilomètres à l'ouest de Domagné, l'établissement d'Ossé La Claraiserie a été fouillé par J. Le Gall en 2013 (Le Gall, à paraître et Barbeau, 2011a, p. 77-87). À l'est de Domagné, l'enclos de Louvigné-deBais, repéré en prospection aérienne par G. Leroux en 2010, a pu être partiellement dégagé lors du diagnostic archéologique (ibid., p. 23-26). L'arasement du site, ainsi que le peu de mobilier, n'ont pas permis de dater précisément l'occupation, qui est néanmoins attribuée à La Tène finale.

Sur la troisième section, deux sites ont été mis au jour sur la commune de Torcé. Le premier, au lieu-dit La Grande Maçonnais 2, a livré un bâtiment sur poteaux du Hallstatt final ou de La Tène ancienne fouillé par Éveha en 2012 (Michel, 2013). Le second diagnostiqué à La Rougerie est un autre exemple de bâtiment sur tranchée de fondation daté de La Tène ancienne ou moyenne (Juhel, 2010, p. 75-80). 

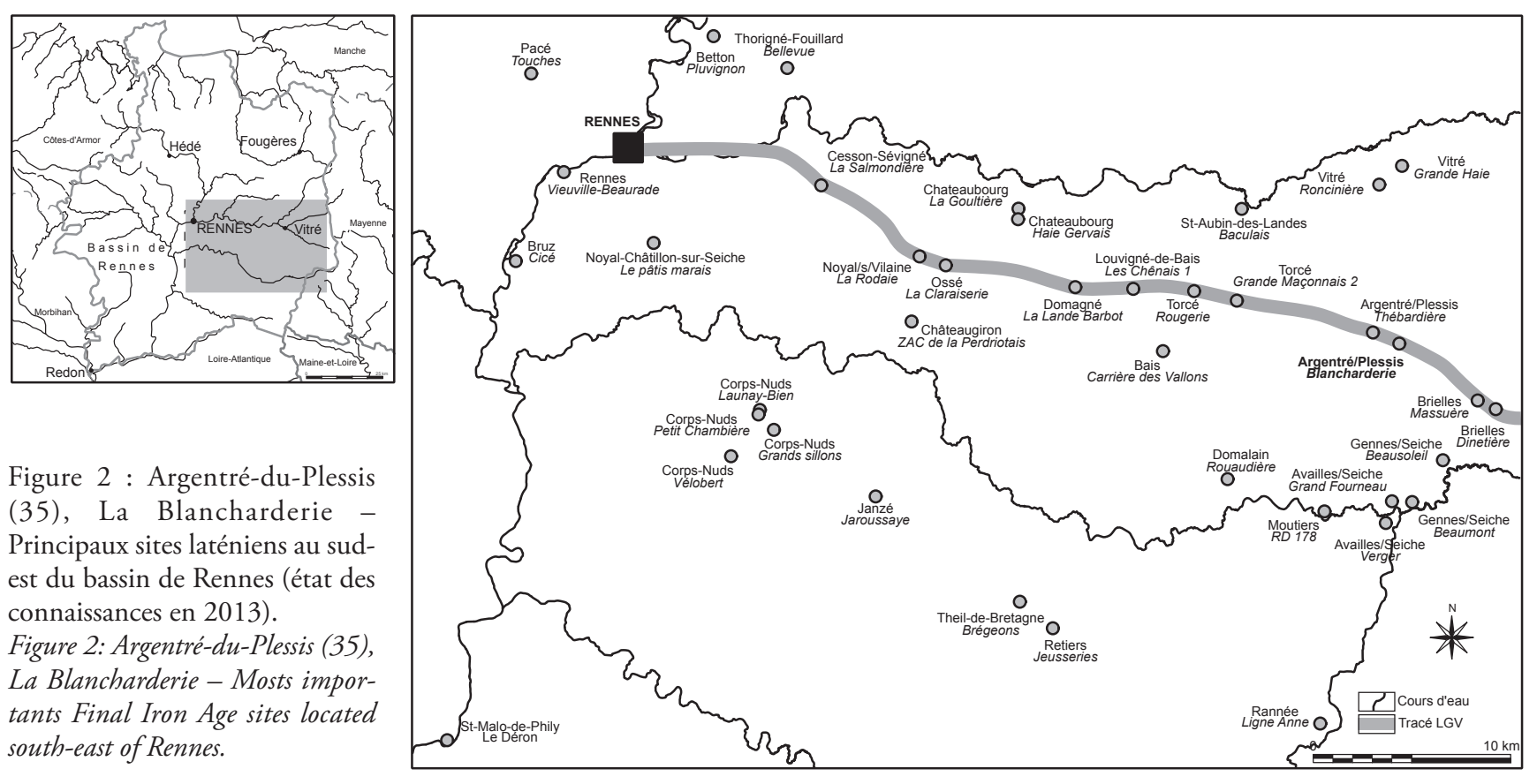

Figure 2 : Argentré-du-Plessis (35), La Blancharderie Principaux sites laténiens au sudest du bassin de Rennes (état des connaissances en 2013).

Figure 2: Argentré-du-Plessis (35), La Blancharderie - Mosts importants Final Iron Age sites located south-east of Rennes.

Enfin à Argentré-du-Plessis la Thébardière, lors du diagnostic, un angle d'enclos quadrangulaire attribuable à la seconde moitié de La Tène a été mis au jour (Juhel, 2010, p. 27-29).

Le site d'Argentré-du-Plessis La Blancharderie se situe sur la quatrième et dernière section de la LGV en Ille-et-Vilaine. Traversant les communes d'Argentré-du-Plessis et du Pertre, le tracé ferroviaire s'implante ici dans un fond de vallée à la confluence de deux ruisseaux d'un affluent de la Vilaine (Toron, 2012). Vers l'est sur la commune de Brielles, aux lieux-dits La Dinetière mais surtout à La Massuère, se développent des occupations rurales occupées à la fin de La Tène et à l'époque romaine. Diagnostiqués en 2010, deux sites ont fait l'objet d'une fouille en 2011 par D. Pouille (Leroux, 2010; Pouille, 2013). Plusieurs autres sites ont par ailleurs été mis au jour sur la suite du tracé parmi les 15 autres sections qui concernent les départements des Pays-de-la-Loire.

\section{L'origine gauloise d'Argentré}

L'établissement rural de La Blancharderie se situe en territoire Riedones, proche de la frontière avec les Aulerques à l'est et Namnètes au sud. La voie antique dite Le chemin des Saulniers, qui traverse la commune d'Argentré-du-Plessis, fait la liaison entre Condate (Rennes) et Vindunum (Le Mans) à la période romaine. Plus au sud, la voie de communication gauloise dite Le chemin des miaules qui traverse la région garde en mémoire la présence de l'activité métallurgique aurifère. D'ailleurs, le toponyme d'Argentré est dérivé d'Argantorate, qui fait référence à l'orpaillage en milieux alluvionnaires (Pailler, 2006). Ce territoire est alors rapproché de l'extraction et de la métallurgie de l'or (Meuret, 1993). L'actuelle commune d'Argentré-du-Plessis est en effet située dans une zone d'étangs et de plusieurs petits cours d'eau dans lesquels l'orpaillage se pratique dès la Protohistoire. Toutefois cette activité ne laisse que peu de traces archéologiques et aucun vestige artisanal de ce type n'a été identifié lors de la fouille de La Blancharderie.

\section{LA MISE EN ÉVIDENCE D'UNE OCCUPATION DE FOND DE VALLÉE}

\section{Organisation générale}

La Blancharderie offre la particularité de se situer en fond de vallée à proximité immédiate de la confluence de deux ruisseaux encore en activité (fig. 3). C'est la spécificité de ce contexte qui a motivé la prescription d'une opération de fouille archéologique, visant à étudier l'articulation entre le milieu naturel et l'installation gauloise.

Le tracé linéaire nord-ouest à sud-est de la LGV n'a permis de mettre en évidence que le bord nord de l'établissement laténien, sur près de $900 \mathrm{~m}^{2}$. Situé à l'extrémité de la dénivellation du plateau, il se caractérise par un système de fossés rectilignes délimitant deux état d'enclos trapézoïdaux. Ces fossés s'étendent à un établissement plus vaste en dehors de l'emprise au sud et à l'ouest. Les départs de fossés secondaires ainsi qu'un petit bâtiment sur quatre poteaux 


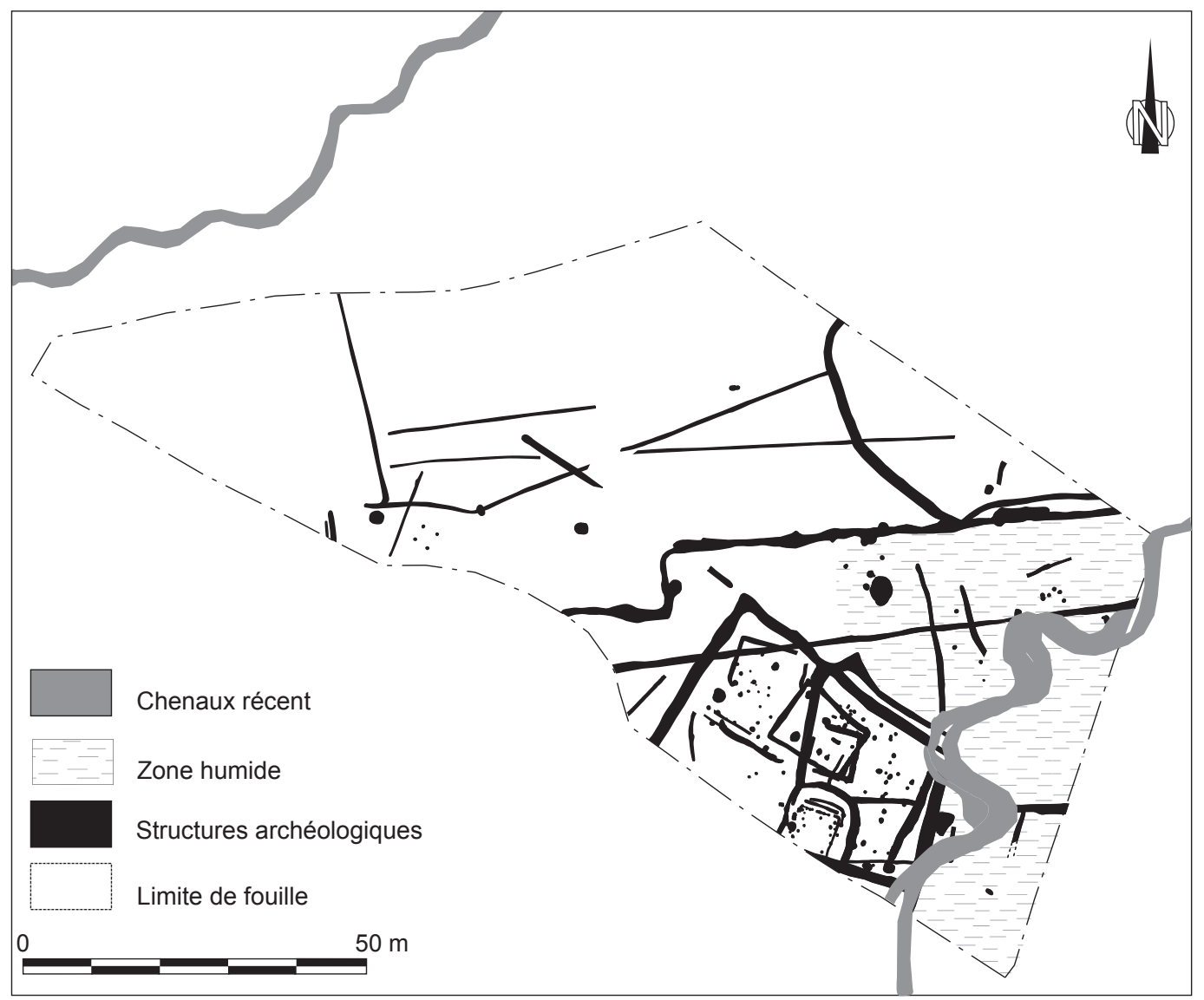

Figure 3 : Argentrédu-Plessis (35), La Blancharderie Plan général du site et des paléochenaux. Figure 3: Argentrédu-Plessis (35), La Blancharderie General plan of features excavated.

hors enclos permettent d'envisager une installation bien plus large qui se développe vers le plateau. Par ailleurs, aucun aménagement d'entrée, ou système de franchissement, n’a été mis au jour sur cette portion d'enclos.

L'une des spécificités de cet établissement réside dans la multiplication des constructions internes qui révèle une succession d'au moins cinq ensembles architecturaux (fig. 5 et 7, Ensemble A à Ensemble F) dans un espace particulièrement réduit. D'une manière générale, ces bâtiments s'organisent parallèlement aux fossés de délimitation des enclos. La chronologie relative entre ces ensembles semble témoigner d'une même volonté d'organisation vers le nord et l'ouest de l'enclos principal. Cette disposition, confortée par des bâtiments systématiquement ouverts vers le sudouest, suggère un axe de circulation dans l'espace interne mais qui échappe toutefois à l'emprise de la fouille.

\section{L'évolution hydro-sédimentaire du site}

L'étude géomorphologique menée par J. Brenot (Géomorphologue, Éveha) a permis d'observer l'intégralité du séquençage de ce contexte hydrographique.

Plusieurs phases successives ont été mises en évidence, la plus ancienne attestée dans le talweg se présente sous la forme d'alluvions grossières de sables et graviers ferrugineux (fig. 4). La plaine alluviale est alors à topographie relativement plane et très large. Ces niveaux alluvionnaires semblent correspondre à la mise en place de chenaux en tresses qui sculptent le bas de pente du vallon à une époque difficile à déterminer. Les quelques tessons ramassés en surface situent tout au plus l'ouverture et le drainage de la zone humide au cours d'une vaste période allant du Néolithique à la Protohistoire ancienne. Seules quelques structures érodées par les dynamiques fluviales témoignent d'un épisode d'installation dans cette zone probablement dès la fin du vi siècle avant notre ère.

La deuxième séquence est une période de diminution de l'activité hydrographique, matérialisée par une épaisse strate limono-argileuse hydromorphe et bioturbée qui succède aux alluvions grossières. Cet épisode entretient le colmatage progressif du talweg, qui prend alors la forme d'une zone palustre. C'est durant cette période que l'établissement gaulois est réellement aménagé sur la bordure du talweg.

L'étude chrono-stratigraphique n'a pu préciser avec certitude la période de fonctionnement de ce marais car les relations entre les dépôts naturels sont trop incertaines en raison de la forte hydromorphie du site. Ce marais est polyphasé avec des périodes d'assèchement qui ont permis 


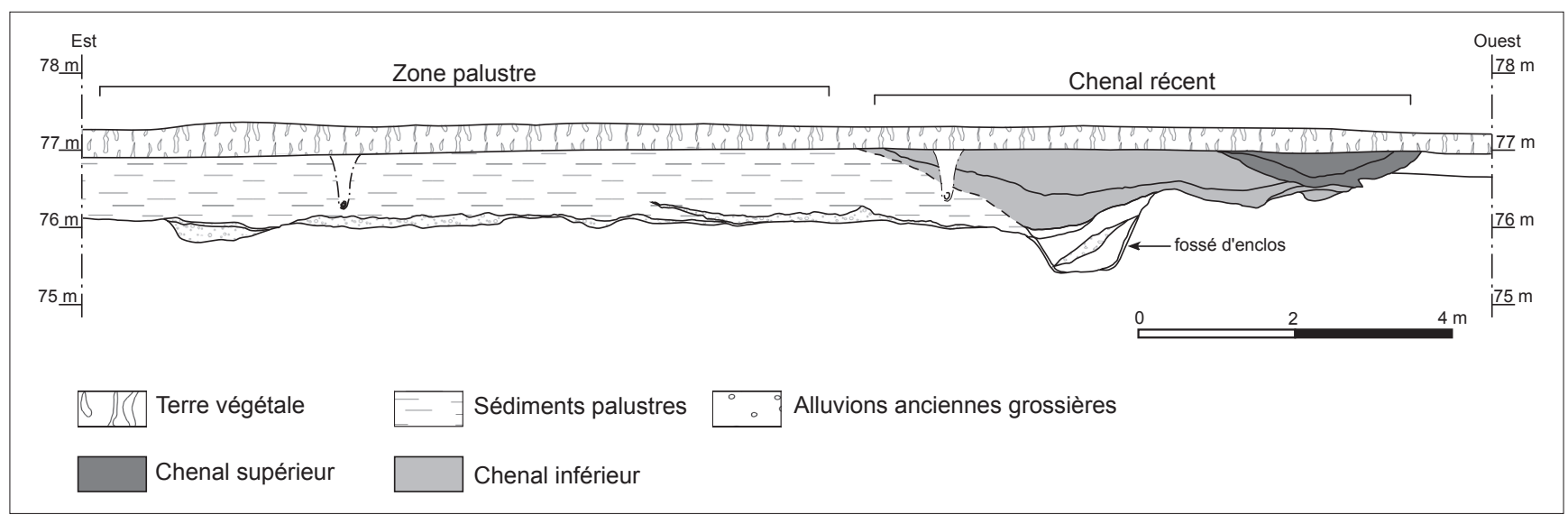

Figure 4 : Argentré-du-Plessis (35), La Blancharderie - Coupe sud à travers la zone humide et le fossé est de l'enclos laténien. Figure 4: Argentré-du-Plessis (35), La Blancharderie - Wetland and ditch enclosure section.

l'installation des gaulois sur la rive gauche du talweg. Les occupants du versant ont alors construit dans une zone qui est déconnectée de la dynamique alluviale. Toutefois on ne peut exclure complètement que la zone palustre soit postérieure à l'occupation principale laténienne et que ses sédiments témoignent d'une avancée de la plaine alluviale sur le versant.

En effet, l'état de paléochenal encore visible sur le cadastre napoléonien du XIX ${ }^{\mathrm{e}}$ siècle est en fait le résultat d'une migration progressive de deux chenaux anciens. L'écoulement de ces derniers se confine sur sa rive gauche, en raison d'une diminution de la dynamique alluviale. Cette phase se développe depuis l'abandon du site laténien jusqu'à l'époque moderne. Ce phénomène de migration des chenaux vers l'est du site va avoir pour conséquence l'érosion progressive et intense des structures archéologiques, et notamment des fossés d'enclos laténien. Ces chenaux seront ensuite canalisés artificiellement à une époque récente.

\section{Première phase (III ${ }^{\mathrm{e}}$-début du $\mathrm{II}^{\mathrm{e}}$ siècle av. J.-C.)}

Entre le $\mathrm{III}^{\mathrm{e}}$ et le début du $\mathrm{II}^{\mathrm{e}}$ siècle av. J-C, la première occupation du site s'implante sur le micro-relief bordier de la zone humide et connaît une activité importante à La Tène moyenne (fig. 5). La zone palustre y est alors probablement active, mais son colmatage progresse déjà vers un probable assèchement. Un système d'enclos est en place à cette période (st 174, st 203 et st 233); le bord du talweg formant une limite naturelle pour l'espace habitable. Il n'est pas possible de savoir si l'enclos trapézoïdal existe sous la forme qui nous est parvenue pour la période suivante. Les données stratigraphiques sont lacunaires sur les fossés de façade est, en raison de l'érosion des vestiges par la migration du chenal récent (st 137 et st 233). Par ailleurs, ces fossés d'enclos étaient probablement entretenus et agrandis tout au long des deux phases d'occupation. Un élément structurant transparaît toutefois sur la façade ouest. Le fossé (st 174), large d'1,20 m pour une profondeur d' $1 \mathrm{~m}$, est agencé en fonction de l'aménagement d'un fossé curviligne (st 153) qui enceint un bâtiment sur tranchée (fig. 5 et 6). Ainsi l'enclos n'est pas strictement géométrique mais semble plutôt fonctionnel avec le bâtiment, voire avec la zone humide au nord. De plus, ces fossés revêtent un caractère particulier avec la présence récurrentes à l'ouest et au nord de surcreusements séparés par des " dos d'ânes " particulièrement bien marqués (fig. 5 et 8 , st 174 et 203).

Il est par ailleurs intéressant de noter que d'autres aménagements de ce type sont connus sur des établissements domestiques de La Tène, comme les « dos d'âne " identifiés sur le site d'Yvré-l'Évêque dans la Sarthe (Vacher et Bernard, 2003, p. 195-196). Ce type d'aménagement pourrait être en relation avec une gestion de l'eau de type drainage et/ou retenue des eaux.

Cette organisation donne une position centrale au bâtiment ovalaire en limite d'emprise qui, de surcroît, livre la majeure partie du mobilier archéologique (céramiques, plaques foyères, outillage lithique, industrie métallurgique, faune...).

\section{Le bâti sur tranchée de fondation à pans arrondis}

L'ensemble A/B est l'unique architecture du site dont le mobilier est clairement rattachable à la fin du $\mathrm{III}^{\mathrm{e}}$ siècle jusqu'au début du II ${ }^{\mathrm{e}}$ siècle avant notre ère (fig. 6). Non seulement son implantation semble privilégiée, mais son aménagement est atypique. Il est cerné par une enceinte fossoyée continue d'un $1,30 \mathrm{~m}$ de large pour $0,50 \mathrm{~m}$ de profondeur (st 153 et st 253). Ce fossé est creusé directement à la base des parois, et connecté au fossé ouest de l'enclos (fig. 5 , 


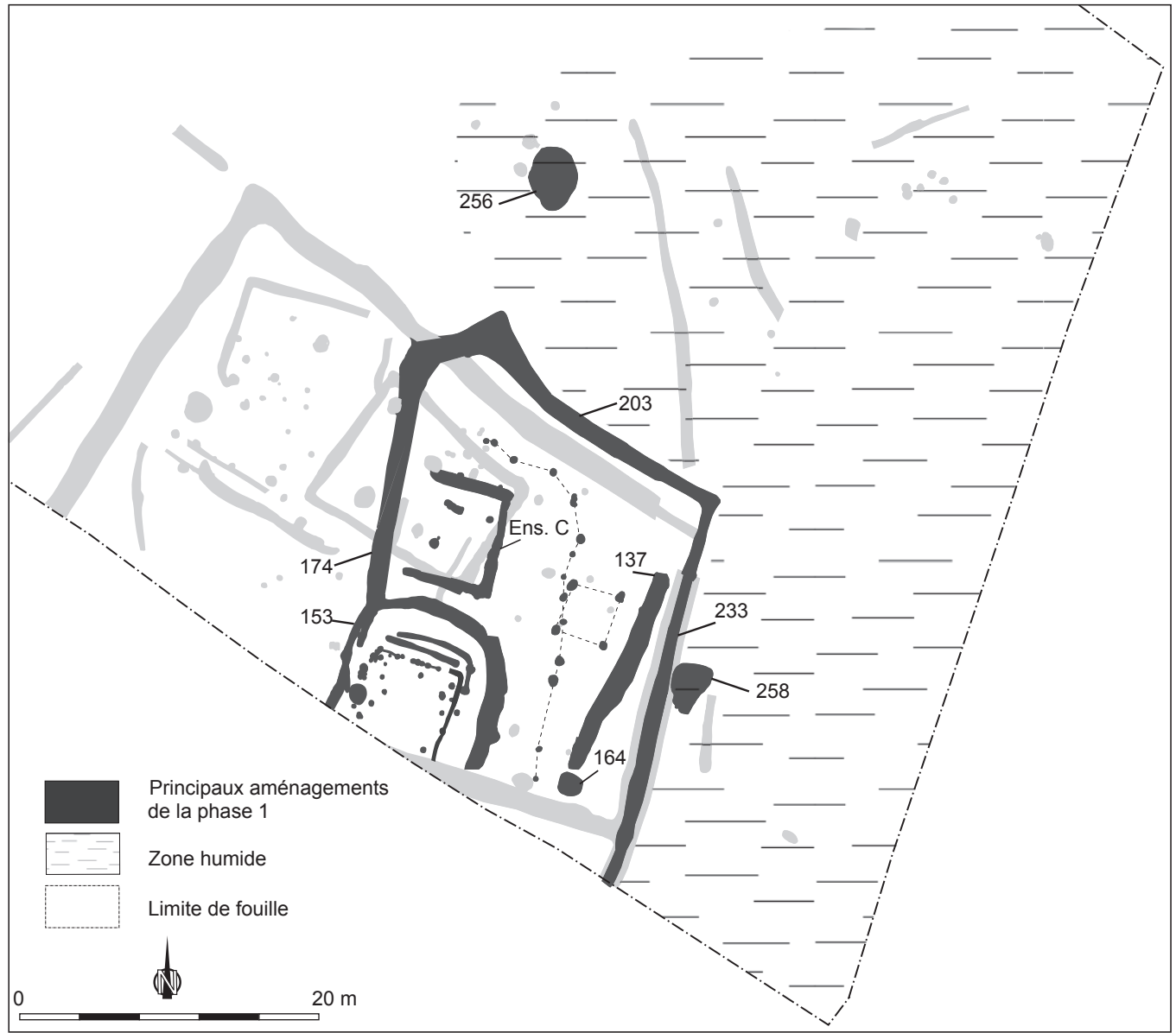

Figure 5 : Argentrédu-Plessis (35), La Blancharderie - Plan des principaux aménagements de la première phase.

Figure 5: Argentrédu-Plessis (35), La Blancharderie - First phase features plan.

st 174). Ce dernier revêt un caractère particulier renforçant l'aspect fonctionnel du système d'enclos et de l'enceinte du bâtiment. En effet, le creusement du fossé ouest, profondément taillé en $\mathrm{V}(1,20 \mathrm{~m}$ par $1 \mathrm{~m})$ présente la particularité d'être segmenté par des dos d'âne (fig. 8). Il est possible d'envisager ici un système de gestion de l'eau autour du bâtiment central, permettant semble-t-il autant le drainage que la captation des eaux de ruissellement.

L'enceinte curviligne (st 153) renferme un unique bâtiment de plan quadrangulaire à pans arrondis, dont probablement la moitié de la surface est hors emprise. Ce bâtiment se distingue par un système de tranchées de fondation multiples, deux segments de tranchée viennent renforcer la paroi nord (st 206 et st 211), elle-même implantée dans une étroite galerie ponctuée de poteaux jointifs (st 192). Ces dernières supportaient probablement les charges de l'élévation, permettant d'éviter l'aménagement de structure faîtière, et ainsi de libérer l'espace interne du bâtiment. Un jeu de sablières hautes et d'entraits devait participer à la charpente soutenant la toiture. Cet ensemble est ouvert à l'ouest où au moins une interruption est renforcée de poteaux (st 189 à st 313). Le mobilier détritique de nature domestique est quasi exclusivement présent dans le fossé curviligne qui ceint le bâtiment (st 153). La proximité de ce fossé avec la paroi suppose une fonction de drainage directement au bas de la toiture.

Le bâti sur tranchée de fondation à pans arrondis et à poteaux jointifs de La Blancharderie coïncide avec la forme des bâtiments connus à partir de la fin de La Tène ancienne et du début de La Tène moyenne dans le nord-ouest de la France. Par ses dimensions, de 6,5 $\mathrm{m}$ de large pour plus de $10 \mathrm{~m}$ de long, ce bâtiment devait atteindre plus de $35 \mathrm{~m}^{2}$ de superficie interne.

Localement un cas isolé, mais très proche, de bâtiment sur tranchée de fondation et poteaux a été mis au jour lors du diagnostic de la section 3 de la LGV sur le site de Torcé $L a$ Rougerie (Juhel, 2010). Ces bâtiments de l'est armoricain se rapprochent des habitations mises au jour par $\mathrm{D}$. Tanguy sur le site de Kerven Teignouse à Inguiniel (Tanguy, 2000). Au moins trois architectures ont une organisation similaire; les restitutions présentant des plans quadrangulaires à poteaux jointifs de 6 à $8 \mathrm{~m}$ d'envergure en moyenne, rattachables également aux $\mathrm{III}^{\mathrm{e}}$ et II ${ }^{\mathrm{e}}$ siècles av. J.-C. (Tanguy, 2000, p. 163). D'autres exemples ont fait l'objet de synthèses régionales tant en Basse-Normandie (Jahier, Vauterin, 2010, p. 119- 
Figure 6 : Argentrédu-Plessis (35),

La Blancharderie - Plan et coupes de l'ensemble A/B. Figure 6: Argentré-du-Plessis (35), La Blancharderie Plan and sections of $A / B$ architectural features.

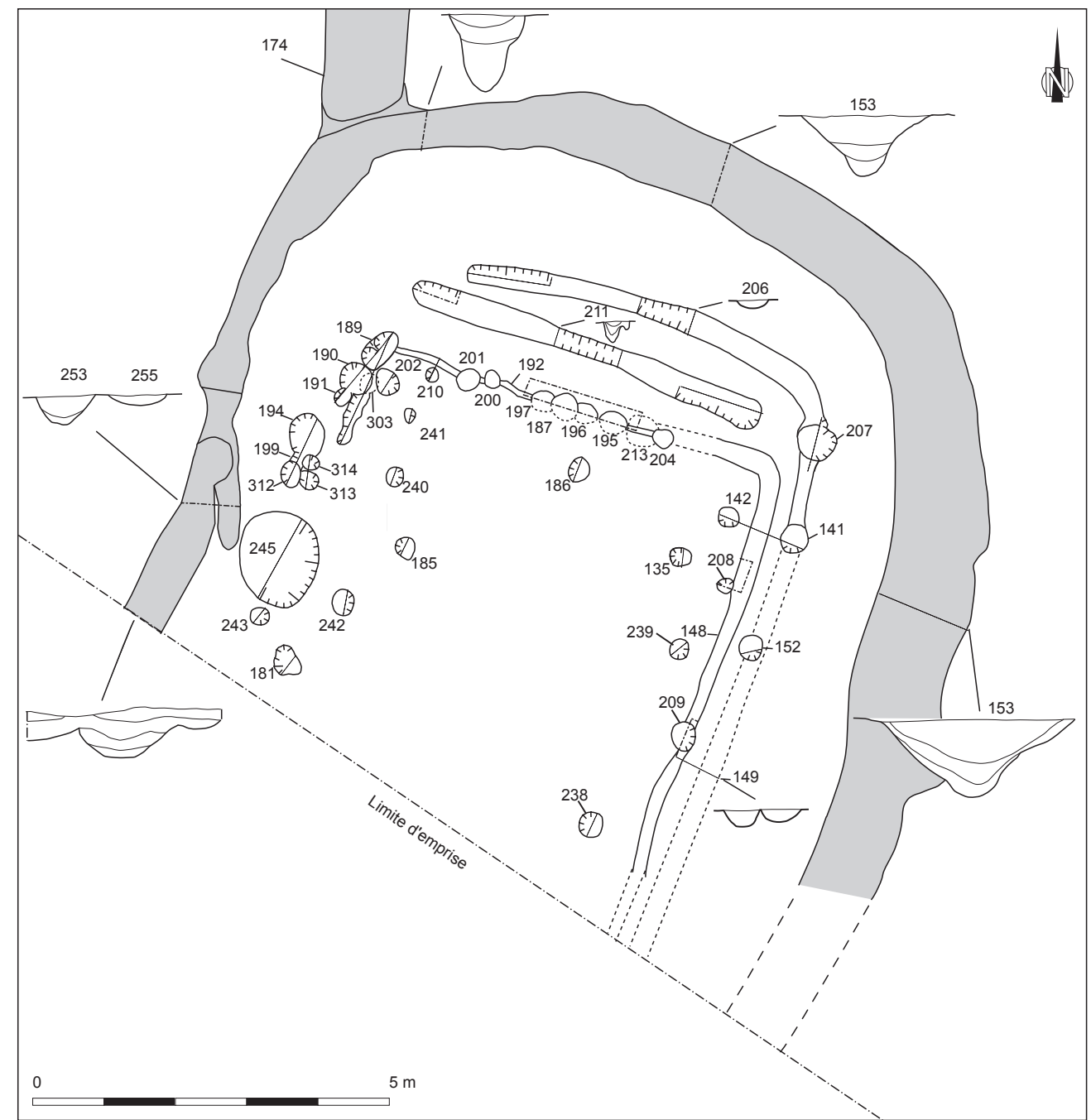

120) qu'en Bretagne et dans les Pays-de-la-Loire (Maguer, Robert 2013). Pour autant, l'architecture à triple tranchée et enceinte périphérique est atypique à La Blancharderie. L'exemple architectural le plus proche typologiquement est le logis de la phase $1 \mathrm{du}$ Camp Saint-Symphorien à Paule en Côtes-d'Armor (Menez, 2008, vol. 2, p. 36). Les dimensions de cet édifice sont certes plus imposantes, toutefois ce dernier associe comme à La Blancharderie une enceinte périphérique renfermant un bâtiment à parois rejetées, aménagées sur tranchées de fondation et poteaux jointifs.

\section{Le bâti sur poteaux}

L'angle nord-est de l'enclos 1 regroupe un ensemble de 30 fosses dont la majeure partie sont identifiées comme des trous de poteau. Les structures circulaires les mieux conservées présentent un diamètre de $0,60 \mathrm{~m}$ à $0,80 \mathrm{~m}$ pour une profondeur de $0,50 \mathrm{~m}$ et un profil évasé à fond irrégulier ainsi que les traces du fantôme du poteau. Les fosses à profils tubulaires bien marqués sont conservées sur $0,30 \mathrm{~m}$ environ pour un diamètre de $0,40 \mathrm{~m}$. Deux aménagements, peutêtre implantés simultanément, sont ainsi suggérés (fig. 5, en pointillé). L'un dont les creusements les plus larges et profonds forment une architecture régulière de type grenier de $3 \mathrm{~m}$ de côté agencée sur quatre poteaux. Le second aménagement est dicté par la linéarité des trous de poteau qui suivent l'angle nord-est de l'enclos primitif. Il pourrait former un dispositif de palissade sur une longueur de 20 à $25 \mathrm{~m}$.

En dehors du bâti, une seule structure fossoyée a pu être associée à cette même phase et au fossé d'enclos est (fig. 5 et 10, st 164). Il s'agit d'une structure de combustion dont le creusement ovalaire et légèrement en sape à fond plat atteint $1,30 \mathrm{~m}$ à $1,80 \mathrm{~m}$ d'envergure pour $0,76 \mathrm{~m}$ de profondeur. Cette fosse présentait à la base des traces de rubéfaction sur les parois, son comblement recelait quelques tessons céramiques mêlés à des fragments d'argile rubéfié et des charbons de bois. Une telle structure pourrait correspondre à un four en fosse ou four semi-enterré. 


\section{Deuxième phase (II siècle-début $\mathrm{I}^{\mathrm{er}}$ siècle av. J.-C.)}

La pérennisation du site au cours du $\mathrm{II}^{\mathrm{e}}$ siècle avant notre ère, est visible par une seconde phase d'aménagement (fig. 7). L'enclos principal se structure sans toutefois ignorer les aménagements précédents, au moins dans un premier temps. La réoccupation majeure du site s'inscrit dans une enceinte grossièrement quadrangulaire d'environ $870 \mathrm{~m}^{2}$ de surface interne. Elle est constituée de quatre fossés (st 129, st 175, st 176, st 233) formant une partition trapézoïdale dont les dimensions avoisinent les $40 \mathrm{~m}$ par $30 \mathrm{~m}$. Ces fossés sont relativement imposants avec $1,80 \mathrm{~m}$ de largeur moyenne et une profondeur constante de $0,90 \mathrm{~m}$. Les profils n'indiquent pas une accentuation particulière de taille et de pendage sur les parties nord et ouest de l'enclos, seul le fossé est (st 233) accuse une pente du nord au sud plus marquée. Par ailleurs, la jonction de ces fossés au sud-est aboutie sous la forme de trois surcreusements séparés par un "dos d'âne " assez marqué, similaire à ceux identifiés sur les fossés de la phase 1 .

Cette partition de l'établissement rural rejoint, par ses dimensions, les exemples régionaux de Retiers (Le Goff, 2003, p. 104-105) ou de Corps-Nuds (Hamon, 2005, p. 71-76), du moins dans leurs premières phases d'aménagements à La Tène moyenne. Il n'est toutefois pas possible de proposer une restitution de l'intégralité de l'établissement puisque les fossés nord-sud (st 175 et st 233) et est-ouest (st 176) échappe en grande partie aux limites de l'emprise du site.

\section{Les bâtiments sur tranchée de fondation}

Au sein de ce second enclos, on dénombre quatre aménagements sur tranchées à angles droits, qui succèdent à la première phase d'aménagement. La première architecture (fig. 5 et 8 , ensemble C), au plan en forme d'agrafe, longe le fossé ouest directement au nord du bâtiment ovalaire. D'après sa position ouverte vers le fossé ouest de l'enclos (st 174), il pourrait être contemporain de la première phase de l'établissement. Il se confond toutefois avec un bâtiment rectangulaire plus vaste (fig. 7 et 8 , ensemble D) qui, a contrario, s'installe sur ce fossé, alors entièrement comblé. Dans l'angle nord-ouest de l'établissement, jouxtant les ensembles $\mathrm{C} / \mathrm{D}$, un autre bâtiment en agrafe (fig. 7, ensemble E) et un bâtiment rectangulaire (fig. 7, Ensemble F), tous deux sur tranchées, sont partiellement conservés. Pour ces derniers

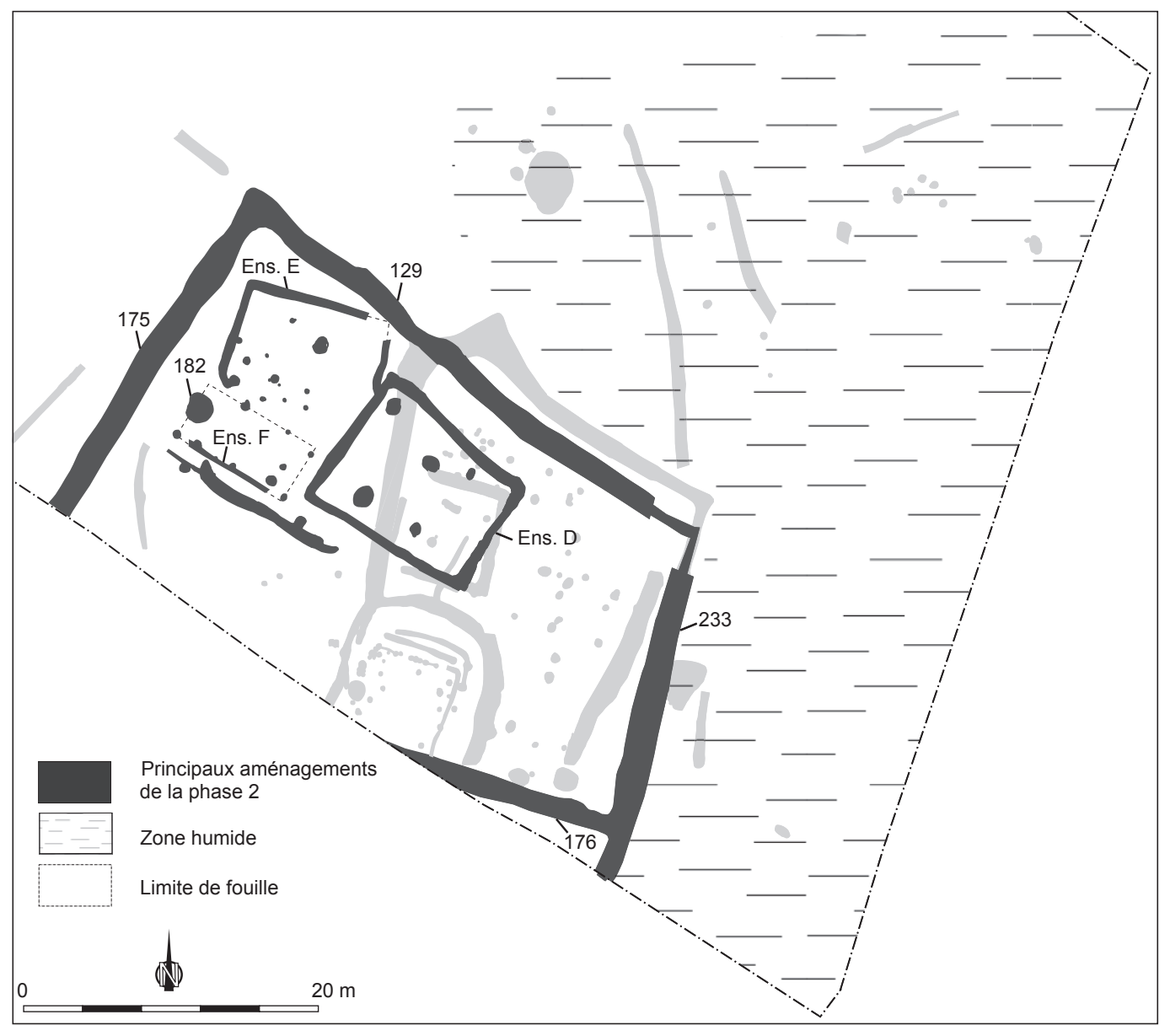

Figure 7 : Argentrédu-Plessis (35), La Blancharderie - Plan des principaux aménagements de la deuxième phase.

Figure 7: Argentrédu-Plessis (35), La Blancharderie - Second phase features plan. 


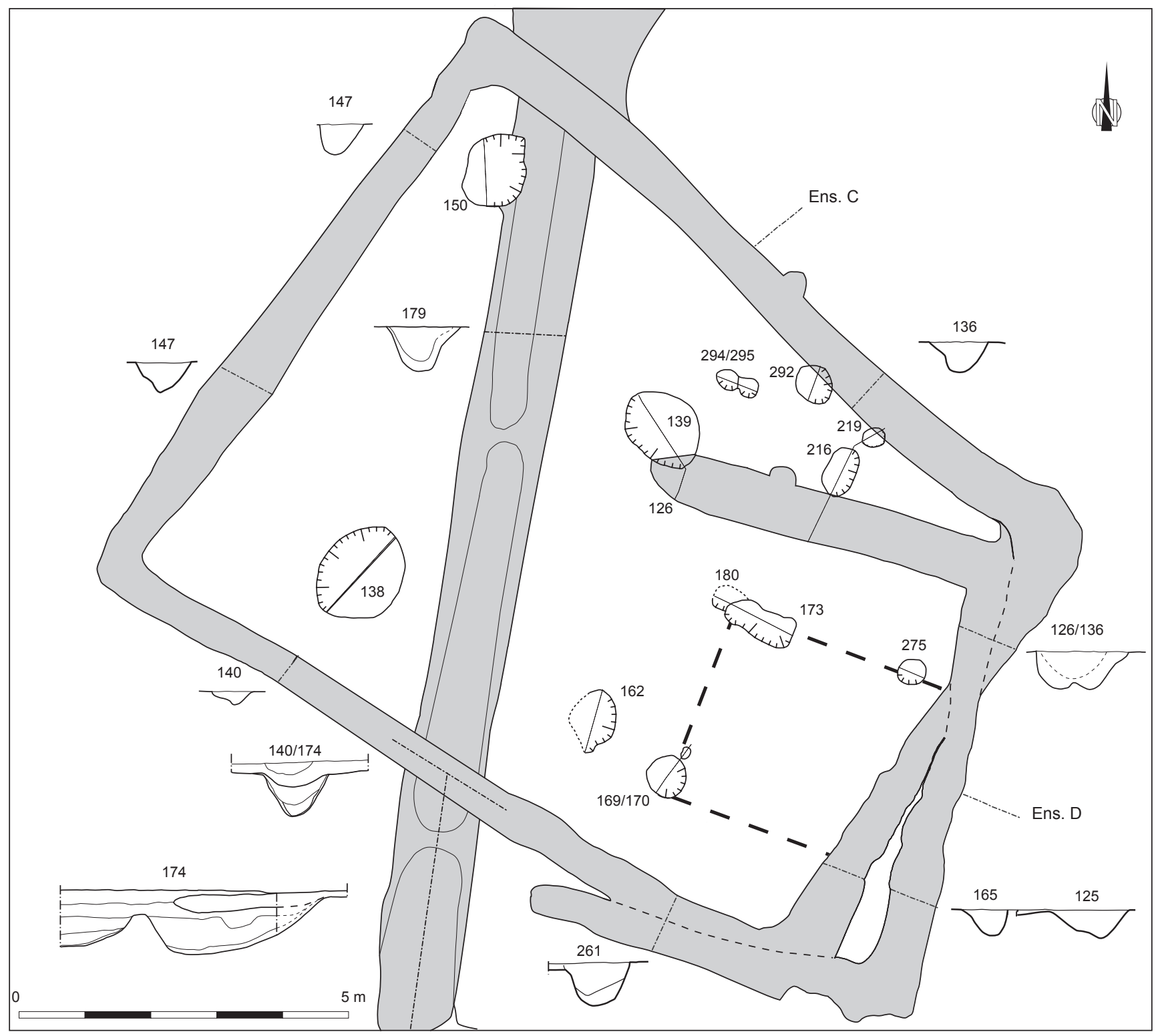

Figure 8 : Argentré-du-Plessis (35), La Blancharderie - Plan et coupes des ensembles C et D.

Figure 8: Argentré-du-Plessis (35), La Blancharderie - Plan and sections of C and D architecturals features.

les éléments datables sont rares mais suggèrent qu'ils appartiennent également à la seconde phase du site.

L'ensemble $\mathrm{C}$ est formé par une tranchée en agrafe mesurant $5,80 \mathrm{~m}$ sur sa façade est et $5 \mathrm{~m}$ sur ses branches disjointes, avec une ouverture à l'ouest (fig. 8, st 125, st 126, st 261). Le creusement de la tranchée, concave à fond relativement étroit, a vraisemblablement servi à l'installation d'une palissade en bois continu. Deux légères cuvettes, interprétées comme des emplacements de poteaux, ont été dégagées dans le prolongement des angles sud-est et nord-est suggérant un renforcement de l'ensemble. La largeur et la profondeur de la tranchée, trop importante $(0,60 \mathrm{~m}$ pour
0,30 $\mathrm{m}$ en moyenne), excluent de fait l'utilisation d'une sablière basse dans le dispositif d'élévation. L'espace interne, d'environ $30 \mathrm{~m}^{2}$, contient au moins trois trous de poteau ayant pu appartenir à la superstructure porteuse (st 169, st 173 et st 275).

Le second ensemble architectural (ensemble D), recouvrant probablement le précédent, est formé lui aussi d'une tranchée peu profonde au profil similaire, $0,5 \mathrm{~m}$ à l'ouverture et $0,30 \mathrm{~m}$ de profondeur en moyenne (fig. 8, st 136, st 140 , st 147 , st 165). Ce bâtiment de forme trapézoïdale à angles droits atteint $12 \mathrm{~m}$ de longueur pour $10,5 \mathrm{~m}$ de largeur sur la plus grande façade au nord-ouest, contre $8,20 \mathrm{~m}$ 
au sud-est. Le creusement est continu et homogène sur les quatre tronçons et aucun système d'entrée n'a été mis au jour. L'aire interne d'environ $90 \mathrm{~m}^{2}$ ne présente pas d'organisation structurée mis à part quelques fosses périphériques (st 138, st 139 et st 150). Un même mode architectural à palissade en bois continu peut être envisagé sur ce bâtiment.

L'ensemble $\mathrm{E}$ présente un fossé peu profond en forme d'agrafe ouvert au sud (fig. 9). Ses dimensions sont supérieures à celles de l'ensemble $\mathrm{C}$ précédemment décrit, la façade nord mesure près de $10 \mathrm{~m}$ de long et la branche la mieux conservée à l'ouest atteint $7 \mathrm{~m}$ de long. Ce plan est donc assez similaire à l'ensemble $\mathrm{C}$, bien que la portance soit plus importante. La largeur du fossé varie de $0,40 \mathrm{~m}$ à $0,60 \mathrm{~m}$, tandis que la profondeur varie d'est en ouest de $0,10 \mathrm{~m}$ à $0,40 \mathrm{~m}$. Le profil affecte par endroits un fond plat assez étroit, indice d'une installation très certainement palissadée. Comme pour les autres ensembles architecturaux d'Argentré-du-Plessis, l'hypothèse d'une sablière basse installée dans les tranchées n'est pas privilégiée. Leur largeur et leur profondeur semblent trop importantes pour la mise en œuvre de ce type de charpente. L'extrémité ouest de l'ensemble E se termine par une inflexion du fossé vers l'intérieur aboutissant à une fosse marquant très probablement l'emplacement d'un trou de poteau en lien avec l'armature du bâtiment. Un amas de terre cuite a été retrouvé, concentré dans cet angle du fossé.

L'aire interne du bâtiment atteint $69 \mathrm{~m}^{2}$ et douze structures fossoyées ont été mises au jour. Toutefois leur agencement ne laisse aucune interprétation possible sur une éventuelle structure porteuse. L'état d'arasement des trous de poteau est par ailleurs très marqué, les creusements étant souvent réduits à de légères cupules de quelques centimètres d'épaisseurs.

Enfin l'ensemble F, directement au sud du précédent, est composé d'un ensemble de structures en creux (fosses, trous de poteau et petit fossé rectiligne) dont l'organisation générale suggère l'existence d'un bâtiment fortement arasé (fig. 9). La tranchée de fondation, partiellement conservée au sud, aurait pu contenir des poteaux dont certains creusements apparaissent relativement bien conservés. Dans la partie nord, sa présence n'a pu être que soupçonnée lors du décapage par quelques concentrations d'inclusions anthropiques dans le substrat. Ce bâtiment de plan quadrangulaire atteindrait des dimensions de 8,80 $\mathrm{m}$ de longueur par $4,50 \mathrm{~m}$ de large soit une surface interne estimée à un peu moins de $40 \mathrm{~m}^{2}$.

La particularité de cet ensemble tient à la présence d'une fosse sur la façade ouest, a priori contemporaine de son fonctionnement (fig. 7, st 182). Cette fosse globalement circulaire, de $2 \mathrm{~m}$ d'envergure pour $0,50 \mathrm{~m}$ de profondeur environ, a livré un exemplaire intact, et peu utilisé, d'une meule active avec encoche. Sa mise au jour légèrement inclinée et surélevée dans le comblement de la fosse, ne permet pas de trancher sur la position primaire de la pièce.

\section{Les fosses-citernes}

Les structures les plus profondes du site de La Blancharderie sont deux larges fosses ovales implantées directement en bor-

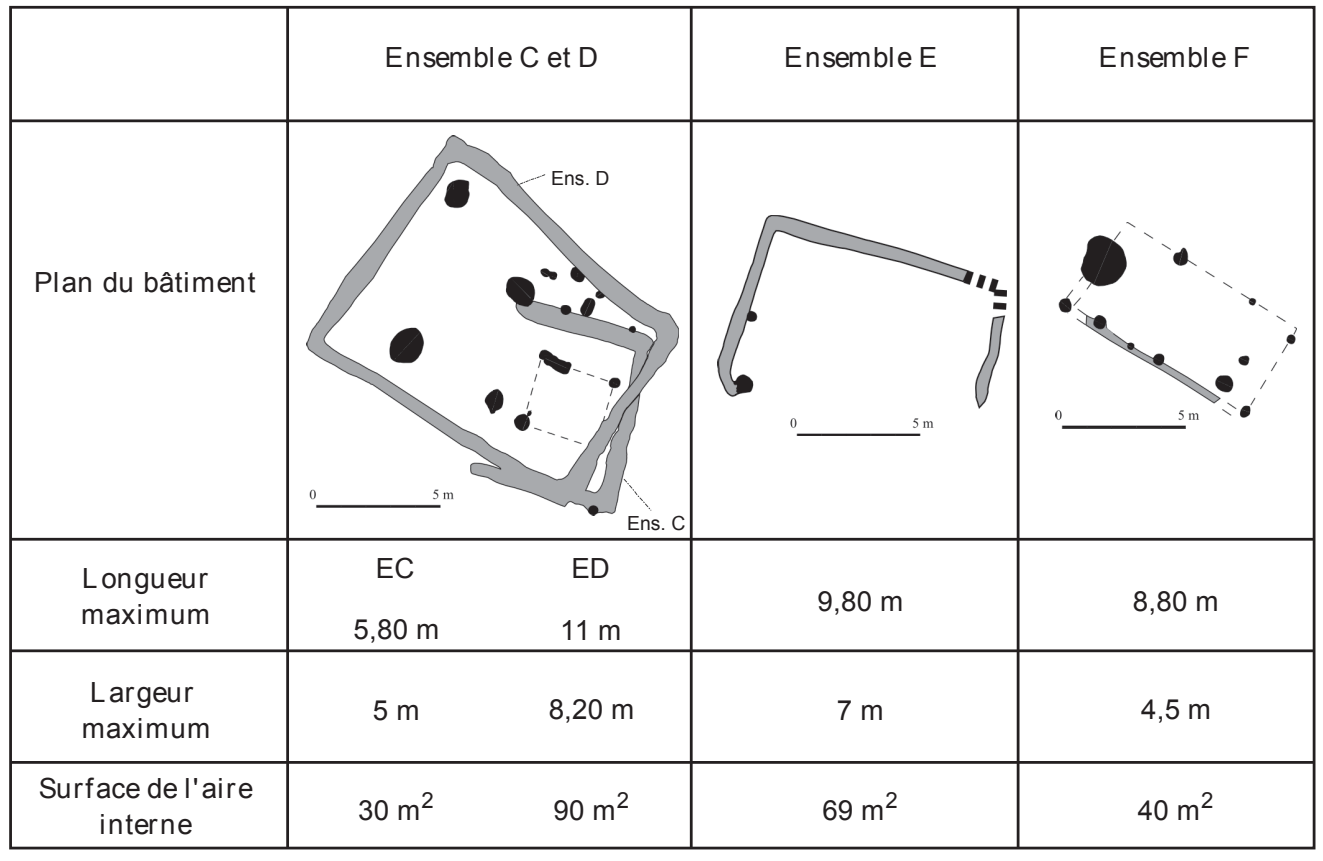

Figure 9 : Argentré-du-Plessis (35), La Blancharderie Plans des bâtiments de la phase 2 et récapitulatif de leurs dimensions.

Figure 9: Argentré-du-Plessis (35), La Blancharderie Summary of plans and dimensions from the second phase bulding. 
dure de la zone humide (fig. 5 et 10, st 256 et st 258). Leur niveau d'apparition au décapage a été perçu sous les limons alluviaux au nord et à l'est de l'enclos.

La fosse 256 au nord atteint $4 \mathrm{~m}$ par $2 \mathrm{~m}$ à l'ouverture et $1,50 \mathrm{~m}$ de profondeur (fig. 10). Le fond circulaire d'environ $1 \mathrm{~m}$ de diamètre présente un comblement d'argile compacte riche en matière organique. Les litages d'argile et d'oxydes de fer qui s'y succèdent plaident en faveur d'un comblement progressif de la fosse. Ces derniers on fait l'objet de deux datations radiocarbones : l'une sur la matière organique marquant le fond de la structure et l'autre dans la partie supérieure du remplissage, directement sous la zone palustre. La première permet de situer le dépôt entre 540 et 400 avant J.-C., soit à la transition entre le premier et le second âge

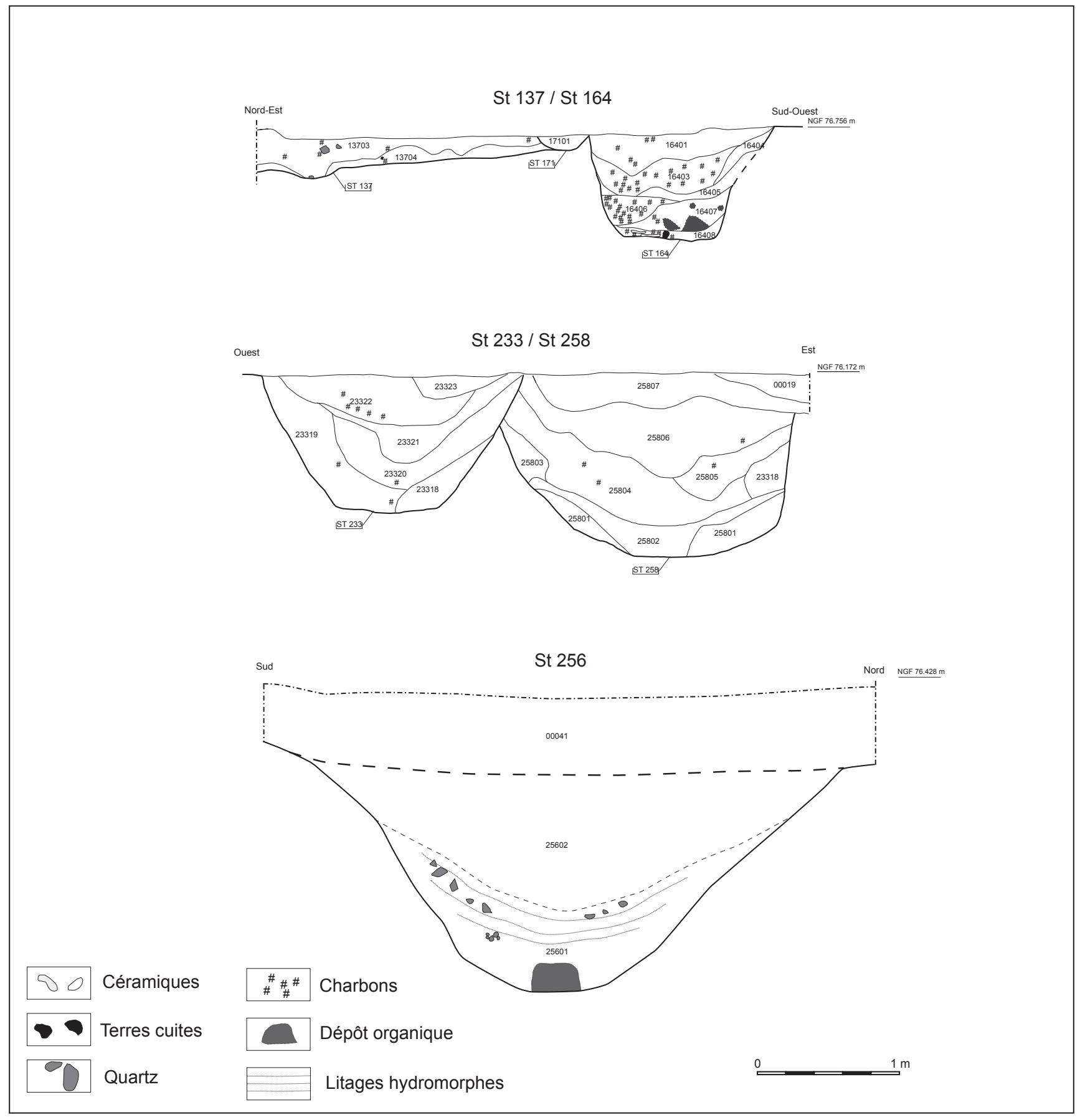

Figure 10 : Argentré-du-Plessis (35), La Blancharderie - Coupes des structures fossoyées.

Figure 10: Argentré-du-Plessis (35), La Blancharderie - Sections of pits features. 
du Fer, tandis que son abandon coïncide avec la première phase d'établissement laténien du $\mathrm{III}^{\mathrm{e}}$ au début du $\mathrm{II}^{\mathrm{e}}$ siècle avant notre ère.

La fosse 258 à l'est, plus ou moins circulaire de $2 \mathrm{~m}$ d'envergure au maximum, atteint presque $1,50 \mathrm{~m}$ de profondeur et se caractérise par un profil évasé à l'ouest et vertical à l'est (fig. 10). Son comblement montre des phénomènes de dépositions sédimentaires progressives, recoupées par le fossé d'enceinte et par le chenal supérieur après son abandon.

La forme des fosses associant larges ouvertures et grandes profondeurs, pourrait correspondre à des fosses à vocation de puisard, c'est-à-dire recueillant d'une part les eaux de pluie mais aussi la remontée de la nappe phréatique. Ce type de structure en périphérie d'une occupation humaine pouvait servir de point d'alimentation en eau. Toutefois la situation en zone humide soulève la question d'une autre fonction de type drainage.

\section{LE MOBILIER}

\section{Répartition générale}

Le mobilier céramique du site est composé de 4435 tessons pour un poids d'environ $50 \mathrm{~kg}$ et un NMI estimé à 493 individus. Aucun fragment d'amphore ou autre importation n'a été retrouvé sur le site. Presque un quart de ces tessons (1 104 tessons pour un NMI estimé à 130 individus) a été retrouvé dans une seule structure à savoir le fossé d'enceinte curviligne à l'arrière du bâtiment A/B (fig. 6, st 153).

De plus, comme pour la céramique, le site a livré $150 \mathrm{~kg}$ de terres cuites essentiellement concentrées dans les fossés de l'établissement, avec une nette concentration du matériel à l'arrière du bâtiment A/B (fig. 6, st 153).

L'étude exhaustive des terres cuites a montré qu'il s'agit essentiellement de plaques foyères, mais également d'autres mobiliers architecturaux. Le matériel lithique présent sur toute la surface du site est relativement riche, mais seulement une vingtaine d'outils a pu être identifiée.

Les restes métallurgiques mais aussi fauniques et organiques sont présents de manières anecdotiques sur le site. Par ailleurs les tests paléoenvironnementaux se sont révélés peu concluant sur les quelques niveaux anthropiques ou naturels potentiellement préservés. Finalement le contexte de forte acidité des sols du massif armoricain ainsi que la forte hydromorphie et l'activité des paléochenaux ont entièrement détérioré ce type de restes archéologiques.

\section{L'assemblage céramique}

L'assemblage céramique d'Argentré-du-Plessis n'a livré que peu de formes complètes, et le corpus des formes est assez réduit. Les principaux types et décors céramiques du site ont été regroupés en planches synthétiques illustrant le vaisselier retrouvé pour les deux phases d'occupation du site.

L'observation macroscopique et à la loupe $(10 \times 18 \mathrm{~mm})$ des pâtes de ces céramiques a permis de les classifier selon différents groupes techniques; essentiellement d'après les types, formes, taille et fréquence de leurs inclusions. Une analyse pétrographique permettrait de déterminer avec précision les types de pâtes employés. Les inclusions les plus fréquemment identifiées au sein de ces types sont les quartz, feldspaths et micas. Ces minéraux présent dans la pâte concordent avec le substrat local issu du massif armoricain. Les pâtes sont essentiellement qualifiées de semi-fines à grossières, excepté un type de pâte fine et triée, qui est utilisé pour certaines formes de jattes ouvertes et basses à profil en esse aux surfaces le plus souvent lustrées.

De nombreuses céramiques présentent des traces de feu sous la formes de tâches noires localisées ou d'encroûtements carbonés. Ces traces pourraient être liées à une utilisation particulière de certains récipients pour la cuisson d'aliments. Toutefois les cuissons de ces vases laténiens sont hétérogènes et essentiellement réalisées en atmosphère réductrice ou avec enfumage en fin de cuisson. Cela rend souvent difficile l'attribution des traces de feu à une réelle trace d'utilisation ou à la cuisson primaire du récipient. Les quelques encroûtements carbonés disponibles sur des récipients très fragmentés du site d'Argentré-du-Plessis ne permettent pas d'envisager une interprétation fonctionnelle satisfaisante pour les vases recélant ce type de traces. De manière plus générale, la position de rejets secondaire détritique des céramiques du site, ainsi que leur fragmentation importante et la quasi absence de formes complètes sur un vaisselier partiel viennent limiter les interprétation fonctionnelles qui pourraient être envisagées sur d'autres types de sites.

\section{Les céramiques de la première phase d'occupation}

Les céramiques issues des structures 153, 137, 211, 174 et le comblement inférieur du fossé 233 sont attribuées à une première phase d'occupation du site (fig. 5 et fig. 11). La caractérisation de cette phase s'appuie sur un total de 1567 tessons, d'un poids de près de $20 \mathrm{~kg}$ et un NMI estimé à 172 individus.

Le corpus des formes est assez peu diversifié. Les formes hautes présentent généralement une une panse assez peu marquée prolongée par une lèvre éversée. Les formes 15396.2 et 15390.1 à lèvre très éversée et panse peu marquée présentent des affinités morphologiques avec un vase du site 


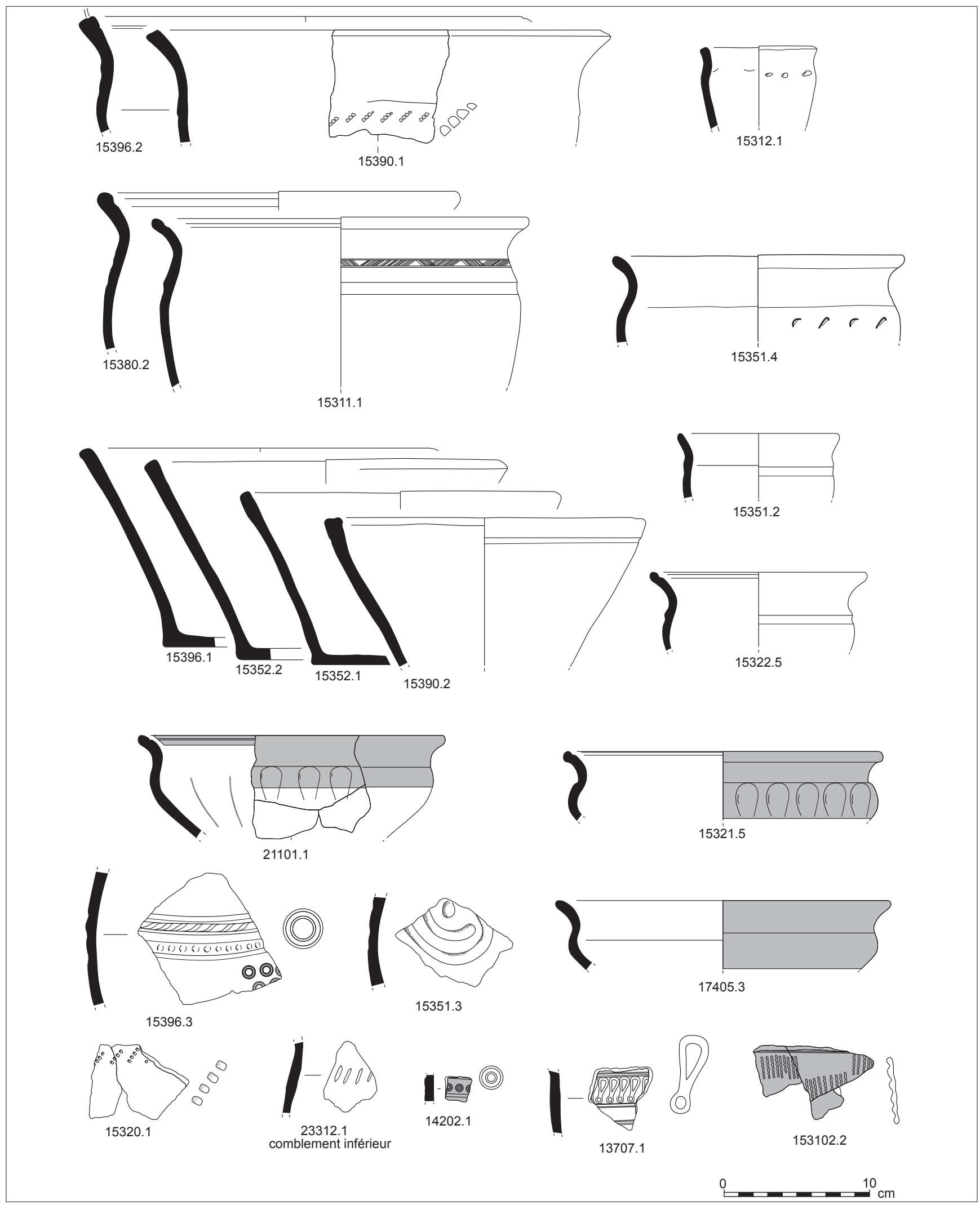

Figure 11 : Argentré-du-Plessis (35), La Blancharderie - Les céramiques de la première phase - III $^{\mathrm{e}}$ s. av. J.-C./première moitié du II ${ }^{\mathrm{e}}$ s. av. J-C.

Figure 11: Argentré-du-Plessis (35), La Blancharderie - The ceramics of the first phase - $3^{\text {th }}$ century B.C.Ifirst half of the $2^{\text {nd }}$ century B.C. 
de Cesson-Sévigné La Salmondière (Durand, à paraitre), F2943) et daté du III $^{\mathrm{e}}$ s. avant J.-C. Ces formes peuvent disposer d'une cannelure labiale interne. Les formes à lèvre éversée et profil en esse plus prononcé 15380.2 et 15311.1 sont identifiées dans le bassin Rennais pour le III $^{\mathrm{e}}$ et surtout le $\mathrm{II}^{\mathrm{e}} \mathrm{s}$. avant J.-C. Elles s'apparentent par exemple à des vases retrouvés sur le site de Vitré, la Grande Haie (Hamon, 2010, pl. 14 et 26) et datées de La Tène moyenne. Ces formes possèdent systématiquement une cannelure encore assez large sur la surface interne de leur lèvre.

Le petit gobelet modelé 15312.1 dont le haut de panse est orné d'une ligne d'impressions sub-circulaires possède de nombreuses concordances régionales pour une période comprise entre la fin de La Tène ancienne et La Tène moyenne, comme sur le site de Bais Carrière des Vallons (Mentele, 2011 fig. 18) où une petite forme analogue est datée entre la fin du IV et le III ${ }^{e}$ s. avant J.-C.

Une série de vases de forme ouverte tronconique (15396.1, $15352.2,15352.1$ et 15390.2) n’apporte pas de réelle précision chronologique mais constitue une composante notable du vaisselier du site.

Les formes basses comportent plusieurs jattes à profil sinueux (21101.1, 15321.5 et 17405.3). Le vase 21101.1 présente un profil en esse assez profond et sa lèvre éversée est munie d'une fine cannelure interne. Sa surface externe est ornée d'un motif embouti en oves ou godrons. L'individu 15321.5, lui aussi décoré d'un motif embouti possède un profil à lèvre éversée pourvue d'une cannelure interne et une panse bien marquée. La jonction entre le col et la panse forme un léger ressaut. La surface externe du vase possède un lustrage abouti ou graphitage conférant à celle-ci un brillant de teinte argentée. Le vase 17405.3 présente quant à lui un profil sinueux continu et sa surface externe est également graphitée.

\section{Le répertoire ornemental présent} sur les céramiques de cette phase est multiple et varié

Les décors estampés prédominent recouvrant une grande variété de motifs. Deux tessons sont ornés de décors d'ocelles, disposées en frise ou en grappe (14202.1 et 15396.3). Ce type de décor d'ocelles en grappe, qui apparaît ponctuellement dès la fin du IV $v^{e}$ s. avant J.-C., caractérise les productions de La Tène moyenne dans l'Ouest de la France. Ce décor orne le plus souvent le haut de panse des vases, comme sur le site de Paule Camp de St Symphorien (Ménez, 2009, fig. 139) daté du second quart du II ${ }^{\mathrm{e}}$ s. avant J.-C.

Le décor estampé de pointillés disposés de biais sur le haut de panse de la céramique est un motif qui se rencontre de manière récurrente sur le site, et principalement sur les céramiques de la première phase $(15390.1,15320.1)$. Un décor similaire se retrouve localement sur un vase du site de Corps-
Nuds, Le petit Chambrière (Hamon et Chérel, 2005, pl. 2) attribué à La Tène moyenne. Un autre décor estampé, en forme de quille ou «aile de paillon ", apparaît sur un tesson issu du fossé 137 (13707.1). Un motifs assez semblable, plus géométrique, se retrouve sur le site de Vitré, La Grande Haie (Hamon, 2010, pl. 2), daté entre la fin de La Tène ancienne et le début de La Tène moyenne, entre la fin $\mathrm{du} \mathrm{IV}^{\mathrm{e}}$ s. et le début du $\mathrm{III}^{\mathrm{e}}$ s. avant J.-C.

Le vase 15351.4 présente une ligne d'impressions de coups d'ongles sur le haut de sa panse et les vases 15312.1 et 15396.3 sont ornés de lignes d'impressions circulaires.

Quelques éléments, principalement des formes ouvertes et basses, possèdent une surface dite graphitée dont le polissage abouti engendre une surface brillante (21101.1, 17405.3 et 15321.5).

Un tesson présente un décor embouti en forme de spirale (15351.3), s'apparentant, dans une forme simplifiée, au style libre des $v^{e}$ et $I v^{e} s$. avant J.-C. Deux jattes à profil en esse sont ornées d'un décor d'oves embouties ou godrons sur la partie supérieure de leur panse (21101.1 et 15321.5). Ces décors emboutis simples se retrouvent localement dans des contextes datés de la fin du IV et du $\mathrm{III}^{\mathrm{e}}$ s. avant J.-C. comme sur le site de Vitré, La Grande Haie (Hamon, 2010, pl. 5) dans un contexte daté de la transition entre La Tène ancienne et La Tène moyenne.

Un individu (15311.1) est orné d'un motif de lignes peignées disposées en frise alternativement de biais, en haut de panse du récipient, souligné d'une strie.

Tous ces éléments convergent vers une attribution chronoculturelle des céramiques de cette première phase centrée sur $\mathrm{La}$ Tène moyenne, entre le $\mathrm{III}^{\mathrm{e}} \mathrm{s}$. et la première moitié du $\mathrm{II}^{\mathrm{e}} \mathrm{s}$. avant J.-C. Les éléments identifiés tendent à indiquer une fréquentation sur le début de la période.

Si peu de formes complètes sont identifiables pour cette phase, les décors sont quant à eux nombreux et diversifiés. Le décor estampé prédomine avec près de 5 types différents : un décor d'ocelles disposées en frise ou en grappe, un décor en frise de pointillés disposés de biais, un second décor en frise de quilles également disposées de biais, et un décor original de lignes ondulées. Plusieurs récipients sont ornés d'impressions circulaires ou de coups d'ongles. Le décor embouti est également représenté avec des motifs d'oves en frise ou de spirale, et un décor peigné est identifiable sur un individu. Quelques récipients possèdent une cannelure ou strie ornant le haut de panse mais ce décor reste assez peu représenté, hormis la présence récurrente de cannelures labiales internes encore assez larges. Enfin, plusieurs éléments de formes, essentiellement des formes basses possèdent un polissage ou graphitage de leur surface. 


\section{Les céramiques de la deuxième phase d'occupation}

Les céramiques issues de l'ensemble C/D (st 147, 140, 125, 136, 246, 157) ainsi que des structures 175, 129, 203 et le comblement supérieur du fossé 233 sont rattachées à une seconde phase d'occupation du site (fig. 7, 12 et 13). Un total de 1180 tessons a été recueilli pour cette phase, d'un poids de près de $13 \mathrm{~kg}$ et un NMI estimé à 127 individus.

Le faciès céramique de cette phase se voit complètement renouvelé avec l'apparition de nouvelles formes et l'appauvrissement des ornementations.

Un vase de très grande contenance (14704.1, fig. 12) présente une forme caractéristique de la fin de la Tène dans l'ouest de la France. Présente sur de nombreux sites du bassin Rennais, cette forme est parfois qualifiée de "proto-dolia " en raison de ses dimensions imposantes. L'exemplaire d'Argentré-du-Plessis est muni d'une gorge creusée sur la surface interne de la lèvre comme sur le site d'Ossé La Claraiserie (Le Gall, à paraître, F524 US 11) et daté du I ${ }^{\text {er }}$ s. avant J.-C.

Plusieurs formes hautes à lèvre légèrement éversée ornées d'un cordon sur le haut de panse (17519.1, 12501.1, 12501.10, fig. 12), ainsi que leur variante à lèvre très éversée et panse striée (12501.2, fig. 12) se retrouvent localement dans des contextes de la fin du $\mathrm{II}^{\mathrm{e}}$ et du $\mathrm{I}^{\mathrm{er}}$ s. avant J.-C. Ce type de forme, très fréquente, se retrouve par exemple sur les sites de Betton Pluvignon (Chérel, 2007, F1319), Bais Carrière des Vallons (Mentele, 2011 F570, fig. 84, F330 fig. 64), Corps Nuds Zac des grands Sillons (Aubry, 2010 -F1009, pl. 4) ou Cesson-Sévigné La Salmondière (Durand, à paraitre, F1401). L'individu archéologiquement complet 17519.1 (fig. 12) possède néanmoins une cannelure labiale interne assez large, critère qui tendrait à indiquer une attribution chronologique peut-être légèrement antérieure.

Quelques jattes à profil en esse sont présentes dans cet assemblage. La forme 13601.1 présente un profil en esse prononcé pour une forme assez profonde. Ces formes de jattes à profil en esse assez profondes caractérisent l'évolution des jattes entre la fin du $\mathrm{II}^{\mathrm{e}} \mathrm{s}$. avant J.-C. et le début $\mathrm{du} \mathrm{I}^{\mathrm{er}}$ s. avant J.-C. dont les profils ont tendance à s'approfondir. Cette évolution est bien visible par exemple sur le site de Bais Carrière des Vallons (Mentele, 2011). La forme 12503.1 est une forme très basse à lèvre en amande et dont la panse possède une rupture en arrête vive. La petite forme ouverte et basse issue du comblement supérieur du fossé 233 (23322.1, fig. 13) possède une lèvre bien dissociée du col $\mathrm{du}$ vase, que l'on retrouve fréquemment dans des contextes de la fin de La Tène en Bretagne, par exemple sur le site de Bais Carrière des Vallons (Mentele, 2011, fig. 86). Ces jattes à profil en esse ne possèdent plus de cannelure labiale interne et le décor graphité est plus rare.
Les formes tronconiques sont représentées par plusieurs exemplaires (13601.2, 13601.3, 24601.1, fig. 13) dont deux vases présentent une cannelure sommitale. Le vase 13601.2 s'apparente aux exemplaires de la phase précédente. La forme 13601.3 est un type qui apparaît localement dans le courant du $\mathrm{II}^{\mathrm{e}} \mathrm{s}$. avant J.-C. et perdure tout au long du $\mathrm{I}^{\mathrm{er}} s$. avant J.-C. Cette forme se retrouve par exemple sur le site de Corps-Nuds Le Petit Chambrière (Hamon et Chérel, 2005, pl. 12) dans un contexte daté de La Tène moyenne ou sur le site de Bais Carrière des Vallons (Mentele, 2011, fig. 73) daté du $\mathrm{I}^{\text {er }}$ s. avant J.-C.

Le corpus des décors pour cette phase est beaucoup plus modeste que pour la phase précédente.

Le décor 14001.1 représente un motif ondé encadré de deux stries. Ce décor ondé est caractéristique de la fin de La Tène et se rencontre durant tout le $\mathrm{I}^{\mathrm{er}}$ s. avant J.-C. dans le bassin Rennais. On peut citer par exemple le site de Bais Carrière des Vallons où il est présent en grand nombre dans des contextes du i ${ }^{\text {er }}$ s. avant J.-C. (Mentele, 2011 fig. 47, 57, 87).

Un décor incisé de biais est localisé sur un cordon en haut de panse d'un vase haut (12906.1, fig. 13).

De nombreux vases hauts présentent un cordon, le plus souvent cerné de deux stries sur le haut de panse ou la jonction $\mathrm{col} /$ panse.

Un décor peigné est visible sur la surface externe de la panse d'un récipient (24601.1, fig. 13) mais il relève plutôt d'un état de surface qu'une réelle ornementation.

Le répertoire des formes de la deuxième phase, quoique plus abondant, demeure assez peu diversifié. Les décors se raréfient et se simplifient, prenant le plus souvent la forme de stries ou cordons.

\section{Les plaques foyères et le mobilier en terre}

Parmi les $150 \mathrm{~kg}$ de terres cuites mis au jour à $L a$ Blancharderie, trois types différents ont pu être distingués en fonction des composantes des différents fragments. Les plus couramment identifiés sont les fragments de plaques foyères qui forment un petit groupe de restes localisés principalement dans l'enceinte à l'arrière de l'ensemble $\mathrm{A} / \mathrm{B}$ (St 153). Dans ce fossé curviligne, un individu a pu être remonté presque intégralement (fig. 14). Il se présente sous la forme d'une plaque rectangulaire à bords arrondis d'une largeur de $47 \mathrm{~cm}$ pour une longueur au moins équivalente. Au moins deux perforations circulaires ont pu être identifiées qui suggèrent un système de suspension. Épaisse de $3 \mathrm{~cm}$ environ elle présente une pâte de facture soignée, où le dégraissant au quartz, bien que présent en quantité importante, se caractérise par sa finesse et son homogénéité. Les traces de rubéfaction, parfois intenses, ainsi que la présence 


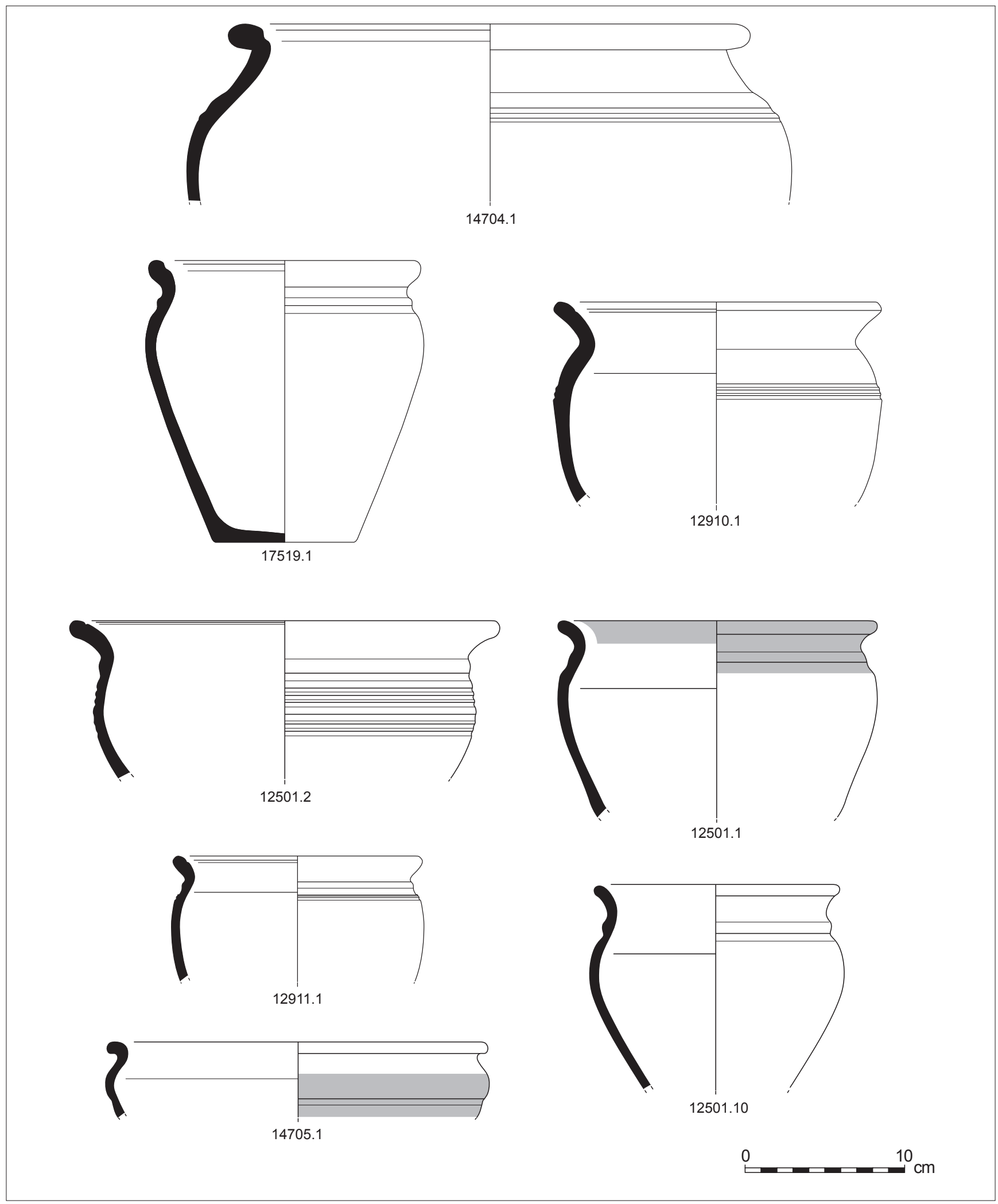

Figure 12 : Argentré-du-Plessis (35), La Blancharderie - Les céramiques de la deuxième phase - deuxième moitié du II ${ }^{\mathrm{e}}$ s. av. J.-C./première moitié du $\mathrm{I}^{\mathrm{er}}$ s. av. J.-C. Formes hautes.

Figure 12: Argentré-du-Plessis (35), La Blancharderie - The ceramics of the second phase - second half of the $2^{\text {nd }}$ century B.C.Ifirst half of the $1^{\text {st }}$ century B.-C. High forms. 


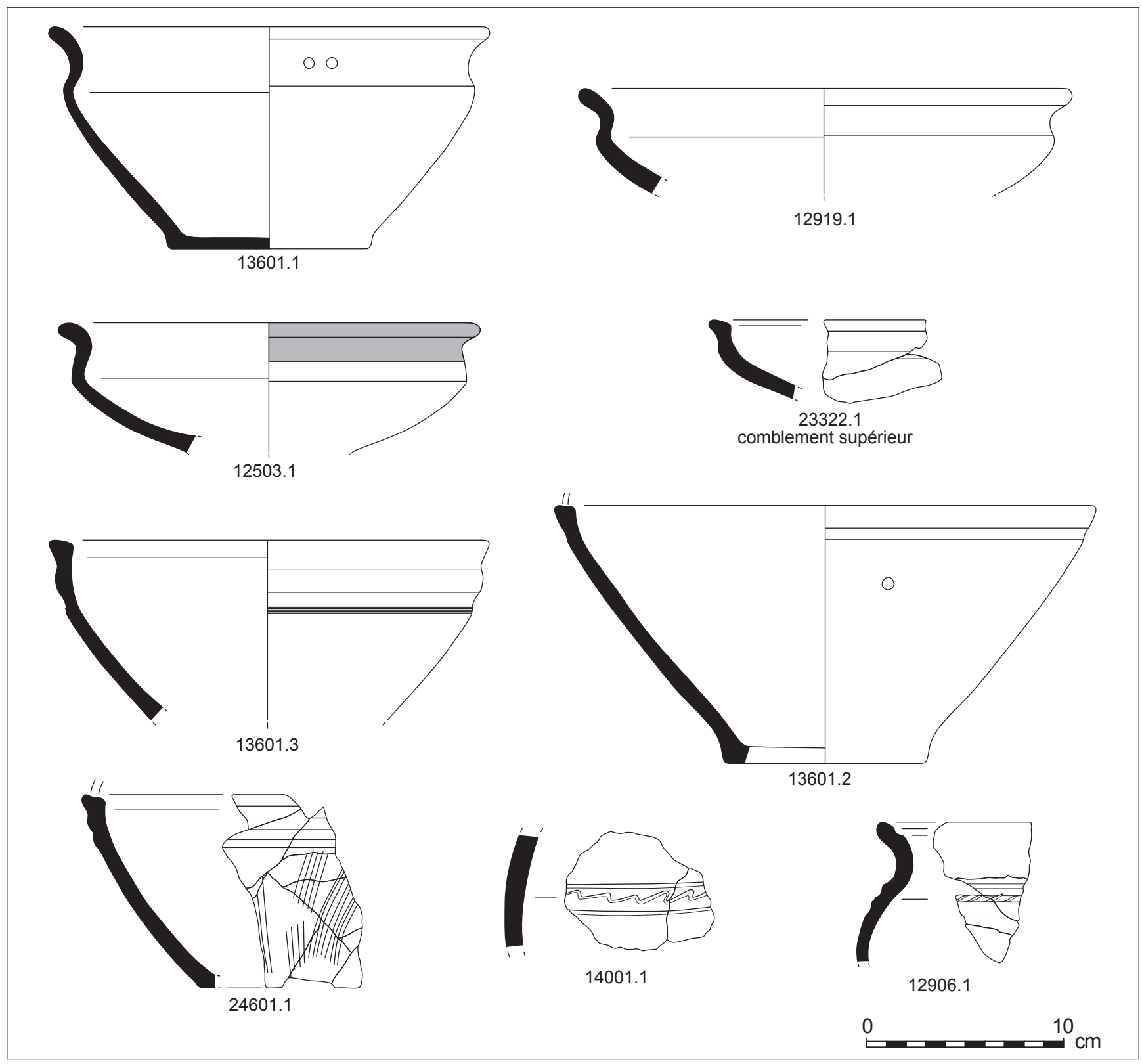

Figure 13 : Argentré-du-Plessis (35), La Blancharderie - Les céramiques de la deuxième phase - deuxième moitié du II ${ }^{\mathrm{e}}$ s. av. J-C./première moitié du $\mathrm{I}^{\mathrm{er}}$ s. av. J.-C. Formes basses et décors.

Figure 13: Argentré-du-Plessis (35), La Blancharderie - The ceramics of the second phase - second half of the $2^{\text {nd }}$ century B.C.Ifirst half of the $1^{\text {st }}$ century B.C. Low forms and decorations.

de suie sur une des surface accréditent l'hypothèse d'une utilisation sur foyer domestique, peut-être amovible.

Les fragments de plaques foyères retrouvées à Argentré-duPlessis sont assez similaires à celles découvertes en Bretagne orientale. Ce mobilier, associé à des utilisations domestiques, est retrouvé fréquemment sur les sites armoricains pour le second âge du Fer (Le Goff, 2003, p. 111).

Cependant le groupe de terre cuites le plus abondant à La Blancharderie est constitué de fragments à pâte grossière, caractérisés par le volume et la taille importante des inclusions de quartz, très hétérogènes dans la matrice argileuse. Les individus appartenant à ce groupe se distinguent par leur épaisseur de 4 à $5 \mathrm{~cm}$ en moyenne jusqu’à $6,5 \mathrm{~cm}$ (fig. 14). Plusieurs concentrations ont été mise en évidence principalement en position de rejet dans les tranchées des ensembles architecturaux (ensemble C/D). La restitution d'une forme, et a fortiori d'une utilisation, est rendue impossible par l'état de conservation de ces restes. Seuls quelques angles et sur- 


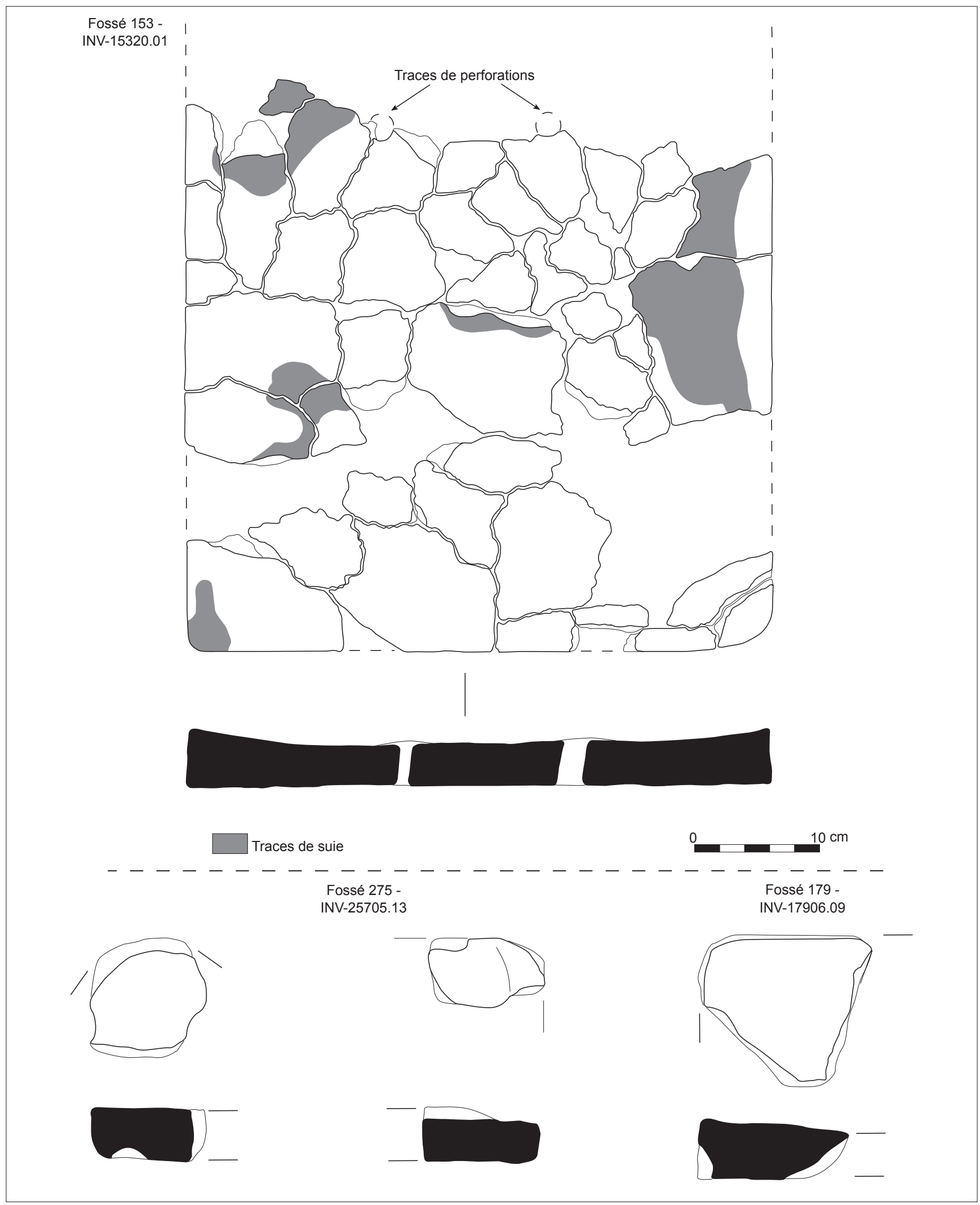

Figure 14 : Argentré-du-Plessis (35), La Blancharderie - Restitution de la plaque foyère, type 1 (en haut); fragments de terre cuite de type 2 (en bas).

Figure 14: Argentré-du-Plessis (35), La Blancharderie - Two different type of hearth slab founded on site. 
faces ont été préservés. En l'absence de tout indice supplémentaire, il est possible d'émettre l'hypothèse de terre cuite à vocation architecturale comme des parois, des fragments de voûtes de fours ou de piles. L'existence de fragments rubéfiés, parfois de manière intense, n'exclut cependant pas d'autres utilisations, il pourrait s'agir d'une variante dans la fabrication des plaques foyères.

Enfin le dernier groupe rassemble indifféremment des restes pulvérulents, sans dégraissant, composés d'un agrégat d'argile, d'oxyde de fer et de schiste trahissant l'utilisation telle quelle, sans transformation, du substrat local pour la fabrication de la pâte. Il s'agit essentiellement de fragments de parois de four et de torchis ayant servi de terre à bâtir.

\section{Le matériel de mouture et l'outillage lithique}

Un peu plus de cent fragments lithiques ont été mis au jour dont vingt-cinq pièces sont déterminées. Malgré un matériel lithique relativement riche, ce site n'a finalement livré que peu d'outils avérés. On peut répartir leurs fonctions en quatre grandes catégories : les éléments de mouture, de polissage, de percussion et les outils sur silex.

Le matériel de mouture est fortement majoritaire avec dix pièces. Il est retrouvé dans le système d'enclos en position secondaire dans les comblements supérieurs des fossés. Trois meules dont un couple meta et catillus ont pu être reconstituées (fig. 15 haut). La troisième a été mise au jour dans une fosse peu profonde à l'ouest de l'ensemble $\mathrm{F}$ (figure 7 , st 182). Ce catillus est remarquable par son poids, près de $40 \mathrm{~kg}$, et son état de conservation intégral. Il ne porte par ailleurs aucune trace d'utilisation (fig. 15, bas). On peut noter l'homogénéité des diamètres des catilli ainsi que de la meta, compris entre $0,35 \mathrm{~m}$ et $0,37 \mathrm{~m}$. Deux des trois catilli possèdent sur leurs flancs des perforations pour permettre le positionnement d'un manchon. Elles sont assez similaires et jamais traversantes.

Le reste du mobilier lithique compte quelques outils de polissage, un percuteur et une petite production sur silex : galet testé, nucléus, éclat, grattoir et lamelle. Hors matériau en silex, quatre types différents de roche ont pu être mis en évidence : granite, grès, quartzite et quartz ainsi qu'une roche sans doute métamorphique mais indéterminée. Le granite est fortement majoritaire dans le macro-outillage mais il faut néanmoins noter la présence de quatre grès assez similaires. De plus quatre fragments de galet en grès, probablement des aiguisoirs ou lissoirs, présentent des surfaces actives avec de grandes plages polies et de rares stries.

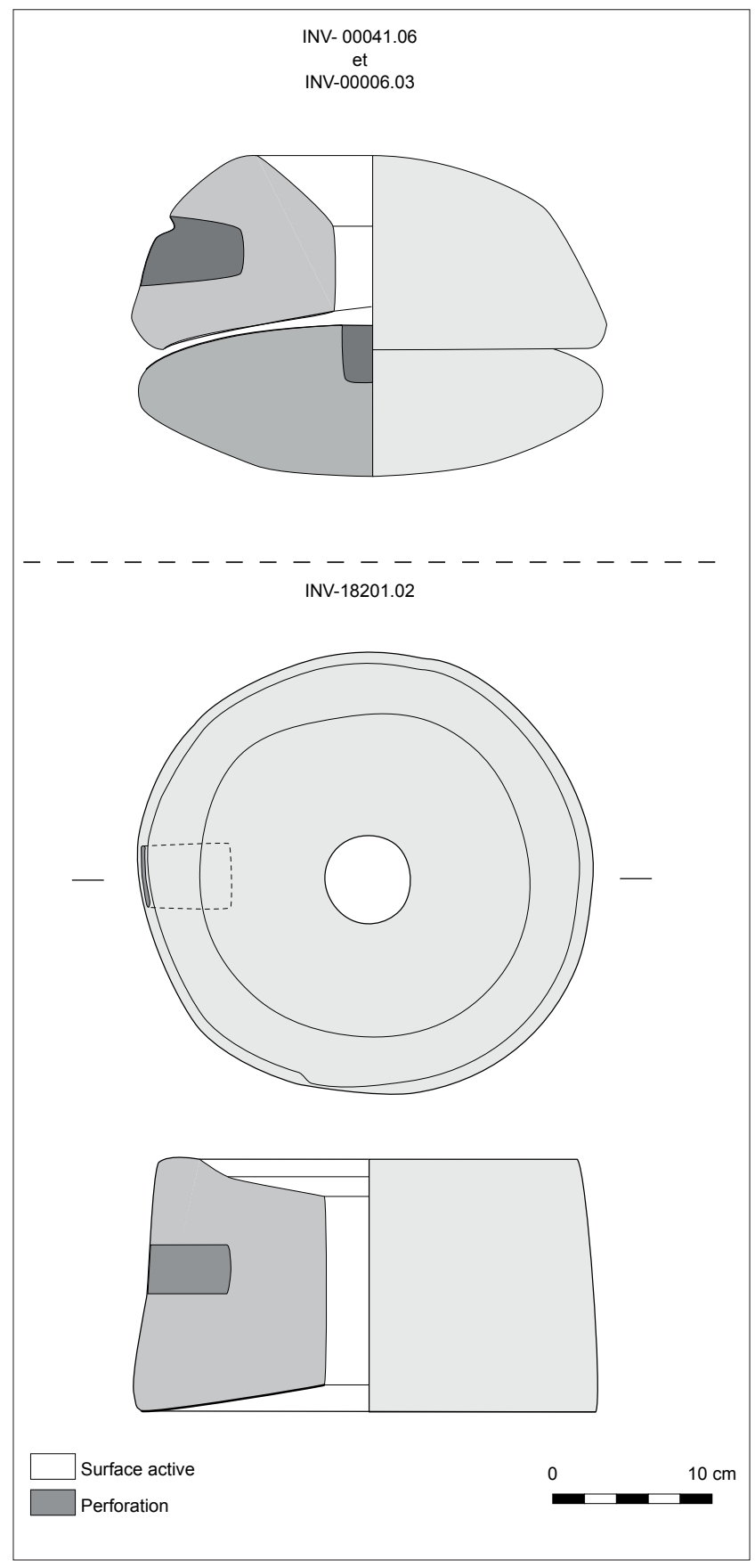

Figure 15 : Argentré-du-Plessis (35), La Blancharderie - Restitution des éléments de mouture découverts sur le site.

Figure 15: Argentré-du-Plessis (35), La Blancharderie-Most important quern founded on site.

\section{Deux éléments de parures laténienne}

Plusieurs fragments d'un même bracelet en lignite ont été mis au jour dans le comblement supérieur du fossé ouest (st 175) du système d'enclos (fig. 16). Il s'agit d'un bracelet d'un diamètre intérieur de $8 \mathrm{~cm}$ et extérieur de $10 \mathrm{~cm}$ envi- 


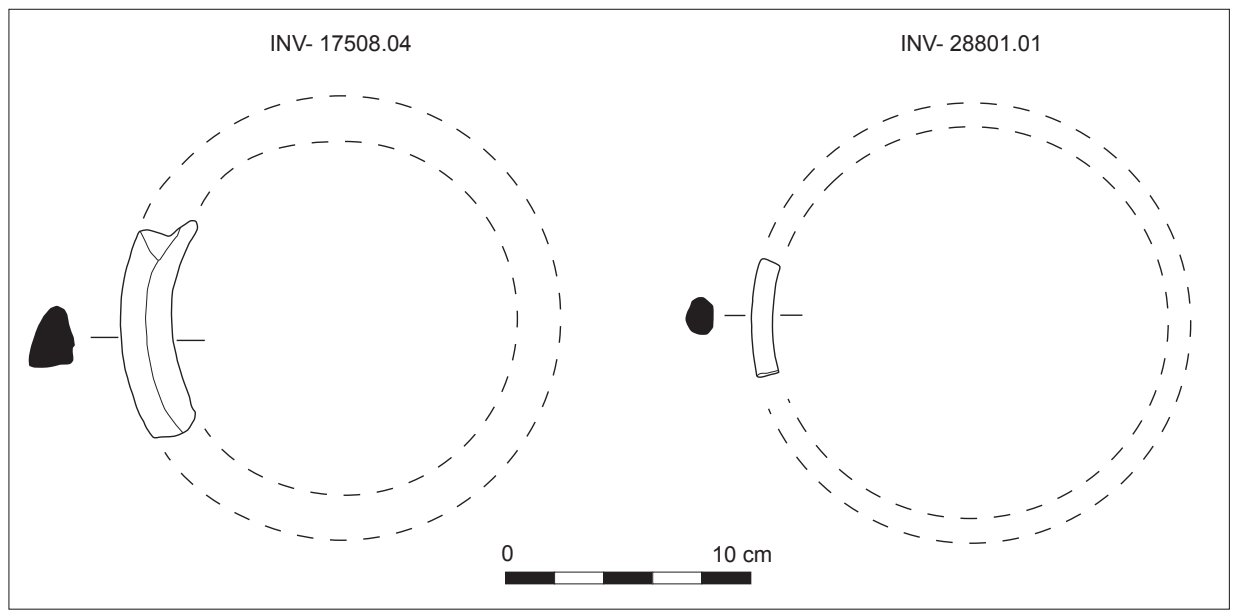

Figure 16 : Argentré-du-Plessis (35), La Blancharderie - Élément de parure en lignite (gauche) et en verre (droite).

Figure 16: Argentré-du-Plessis (35), La Blancharderie - Piece of lignite and glass bracelets founded on site.

ron. De telles dimensions laissent à penser qu'il était porté au poignet par un adulte. De profil triangulaire ou en $\mathrm{D}$, sa section est plane à l'intérieur, et bombée à l'extérieur; son épaisseur maximale est de $0,9 \mathrm{~cm}$. Sa surface est lisse, sans trace de mouluration ou de décor.

Un seul élément en verre a été découvert sur le site, provenant du comblement unique d'un trou de poteau dans le secteur nord-ouest des ensembles E et F (fig. 7 et 16). Le fragment, long d'environ deux centimètres, appartient à un bracelet constitué d'un jonc unique à profil en $\mathrm{D}$, de teinte ambrée, dénué de décor. Il est épais de $0,5 \mathrm{~cm}$ tandis que son diamètre interne peut être estimé à $8 \mathrm{~cm}$, ce qui permet de supposer que le bracelet était porté au poignet par un individu adulte. Il est comparable au fragment de bracelet en verre découvert dans la résidence aristocratique de Saint-Symphorien à Paule (Côtes-d'Armor), dans un niveau d'incendie daté de La Tène C2 (Menez, 2009, p. 302-305).

\section{ConClusion eT DisCussion}

\section{Synthèse des données}

L'établissement mis au jour n'offre qu'une vision partielle d'un habitat enclos qui évolue en continu du début du $\mathrm{III}^{\mathrm{e}}$ siècle jusqu'au début du $\mathrm{I}^{\mathrm{er}}$ siècle avant notre ère. Cette occupation est marquée par une première réelle structuration d'enclos à la fin du III ${ }^{\mathrm{e}}$ ou au début du II $^{\mathrm{e}}$ siècle avant J.-C. Cet état est ensuite réaménagé par un enclos plus marqué, mais suivant l'organisation de la phase précédente, jusqu'au début du $\mathrm{I}^{\text {er }}$ siècle avant J.-C.

Il est dès lors difficile d'évaluer la taille de l'établissement ni même son évolution chronologique. Il s'y développe sûrement un site conséquent pour le second âge du Fer voire au début la période romaine plus en amont sur le plateau. L'environnement spécifique, en bordure de zone humide d'un fond de vallée, joue un rôle dans l'organisation de cette partie de l'établissement rural mais ne semble pas refléter d'activités domestiques ou artisanales particulières. La présence d'une zone humide forme une limite naturelle pour l'habitat, les fossés d'enclos épousant la topographie bordant le talweg. Celui-ci se comble progressivement et connaît des épisodes d'occupation, peut-être de drainage ou d'installations périodiques. Cet environnement humide pourrait également expliquer la présence dans le fossés d'enclos de surcreusements séparés par des "dos d'âne ". Ces aménagements, également observés sur l'établissement de fond de vallée d'Yvré-l'Évêque dans la Sarthe (Vacher et Bernard, 2003), suggèrent que les dispositifs d'enclos participent à la gestion de l'approvisionnement et de l'évacuation de l'eau sur l'établissement.

L'aire interne des enclos de La Blancharderie diffère des établissements de plaine par la densité des aménagements, présentant très peu de chevauchements, et une succession assez nette de constructions peut s'esquisser. Cet agencement induit une importante contrainte spatiale qui a peut-être eu un impact sur l'architecture des bâtiments. La principale caractéristique du bâti est l'aménagement sur tranchées de fondation. Les deux phases d'occupations montrent une tendance évolutive du plan à pans arrondis et poteaux jointifs vers des plans quadrangulaires avec des fossés plus larges et des architectures sur poteaux plantés. Toutefois pour ces derniers, leur structuration interne non cohérente et l'indigence du mobilier posent des questions quant à la restitution et la fonction de tels bâtiments. Les dimensions varient amplement pour couvrir des superficies de $30 \mathrm{~m}^{2}$ à $90 \mathrm{~m}^{2}$.

Ces architectures laténiennes renvoient à de nombreux parallèles régionaux et extra-régionaux tant sur les établissements proches de Vitré La Grande Haie (Hamon, 2010) ou Saint-Sauveur-des-Landes (Sicard, 2010), qu'en Normandie et dans les Pays-de-la-Loire. La forme architecturale à pans arrondis évoluant vers l'enclos quadrangulaire est un jalon 
récurrent sur les établissements du second âge du Fer en France septentrionale.

Le mobilier céramique mis au jour est caractéristique tant dans ses proportions, son état de conservation que dans ses formes, d'un site d'habitat du bassin Rennais pour La Tène moyenne et La Tène finale. Le mobilier quoique peu abondant de la première phase datée de La Tène moyenne vient enrichir le corpus assez limité des assemblages de cette période dans le bassin Rennais. Les céramiques de la seconde phase datée de La Tène finale se conforment aux nombreux assemblages identifiés pour ce contexte chronologique bien documenté.

\section{Un habitat de fond de vallée : un choix d'implantation justifié?}

L'implantation d'un d'habitat en bordure de zone humide de fond de vallée est cohérent avec ce que nous savons, à ce jour, de l'évolution de l'habitat gaulois en France septentrionale (Menez, Lorho, 2013, p. 173-174). Bien que ce type d'installation soit beaucoup moins fréquent que les établissements ruraux positionnés sur des points hauts, les recherches récentes tendent à relativiser leur caractère exceptionnel. Dans l'Oise, par exemple, les micro-reliefs de fonds de vallée sont communément occupés au début du second âge du Fer; ils évoluent progressivement à partir du III $^{\mathrm{e}}$ siècle avant J.-C. vers les plateaux, où la colonisation d'espaces plus vastes sur les hauteurs devient ensuite majoritaire à la fin de la période (Durant et al., 2006, p. 46-51). Un tel schéma d'évolution est envisageable sur le site de $L a$ Blancharderie, et est certainement plus fréquent en Bretagne malgré le faible nombre de découvertes de sites de fond de vallée à ce jour.

Probablement dès la seconde moitié du vi siècle et jusqu’à la première moitié du $\mathrm{I}^{\text {er }}$ siècle avant notre ère, l'occupation basse de La Blancharderie correspondrait à un lieu d'installation privilégié. Les quelques rares vestiges conservés de la fin du premier âge du Fer au début de La Tène attestent ainsi d'une colonisation ancienne du fond de vallée qui va perdurer jusqu'à la fin de l'âge du Fer.

Durant l'occupation principale entre le $\mathrm{III}^{\mathrm{e}}$ et la première moitié du ire siècle avant J.-C., le paysage du site doit être envisagé comme un paysage de rive, mais néanmoins clairement déconnecté d'un chenal, pourtant proche et aisément accessible. L'occupation, caractérisée par de nombreuses unités d'habitations, est implantée sur un micro-relief surélevé, à l'abri de la montée des crues. Ce même schéma d'implantation a été mis en évidence sur le site de SaintMalo-de-Phily (35) où se succèdent trois enclos à La Tène sur la rive occidentale de la Vilaine (Le Goff, 1999). Sur le site de Vieuxville-Beaurade, à Rennes, l'occupation gauloise est, là aussi, implantée en bordure de la Vilaine, à proximité d'une cuvette marécageuse (Leroux, 1993).

A la fin du $\mathrm{I}^{\text {er }}$ siècle avant notre ère, le fond de vallée est délaissé et l'occupation se développe très probablement vers des espaces viables sur le versant et le plateau au sud-ouest.

La confrontation des données anthropiques et environnementales n'a pas encore fourni de réponses quant à cet état de fait. Il peut s'agir à la fois de l'influence d'une péjoration climatique et aussi d'un réel choix des populations qui privilégient les larges espaces cultivables au détriment des zones exigües, plus impropres à l'agriculture. Les problématiques de rythmes d'emprises et de déprises agricoles à l'âge du Fer doivent être abordées à l'échelle d'un terroir plus vaste dans lequel l'occupation d'Argentré-du-Plessis trouverait un écho certain.

\section{Bibliographie}

Aubry L., 20 o - Une exploitation agricole de la fin de l'âge du Fer, Corps Nuds, (Ille-et-Vilaine), RFO - Fouille archéologique, Rennes, SRA Bretagne/Inrap Grand-Ouest, 146 p.

Chérel A.-F., 2007 - "Étude de la production céramique de l'âge du fer ", in Blanchet S. (dir.), Betton "Pluvignon", Ille-et-Vilaine, Vol. 2 "De l'àge du Fer au Moyen-Age ", RFO - Fouille archéologique, Rennes, SRA Bretagne/Inrap GrandOuest, 280 p.

Durand J.-C., (à paraître) - Les occupations protohistoriques et historiques de Cesson-Sévigné (Ille-et-Vilaine), La Salmondière, RFO - Fouille archéologique, Rennes, SRA Bretagne/Inrap Grand-Ouest.

Durand M., Malrain F. et Pinard E., 2006 - «Les sites laténiens de la moyenne vallée de l'Oise du v viècle au $\mathrm{I}^{\mathrm{er}} \mathrm{s}$. avant notre ère : contribution à l'histoire de la société gauloise ", Revue archéologique de Picardie, Numéro spécial 23, 268 p.

Hamon A.-L., 2010 - Vitré, Ille-et-Vilaine, Boulevard de Laval. La ferme laténienne de La Grande Haie, RFO - Fouille archéologique, Rennes, SRA Bretagne/Inrap Grand-Ouest, 243 p.

Hamon A.-L., et Chérel A.-F., 2005 - Corps-Nuds (35). Le Petit Chambière. Un enclos d'habitat du second âge du Fer, RFO Fouille archéologique, Rennes, SRA Bretagne/Inrap GrandOuest, 118 p.

Jahier I., Vauterin C.-C. et Bouvet J.-P., 2010 - "Formes et composantes de l'habitat à l'âge du Fer en Basse-Normandie : architecture, chronologie, organisation, statut - un premier bilan ", L'âge de fer en Basse-Normandie: gestes funéraires en Gaule au second âge du fer : actes du XXXIII colloque international de l'AFEAF (Caen, 20-24 mai 2009), Annales littéraires de l'université de Franche-Comté, 883 Presses universitaires de Franche-Comté, p. 95-138. 
Juhel L., 2010 - Louvigné-de-Bais, Torcé, étrelles, Argentrédu-Plessis, Ille-et-Vilaine, LGV-Secteur 3. Occupations du Néolithique à l'époque moderne sur le tracé de la LGV Rennes-Le Mans, RFO - Diagnostic archéologique, Rennes, SRA Bretagne/Inrap Grand-Ouest, 233 p.

Le Gall J., (à paraître) : L'occupation de la fin de l'âge du Fer et du début de l'époque antique de l'établissement rural d'Ossé (Ille-etVilaine), La Claraiserie, RFO - Fouille archéologique, Rennes, SRA Bretagne/Inrap Grand-Ouest.

Le Goff E., I 999 - Saint-Malo-de-Phily "Le Déron - Carrière des Menais " (35), DFS de sauvetage urgent, Rennes, SRA Bretagne, AFAN, 49 p.

Le Goff E., 2003 - "Armorique et Bretagne " : réflexion sur l'assimilation de ces concepts pour la fin de l'âge du Fer, Revue archéologique de l'Ouest, suppl. 10 [MAndy B. et DE SAulce A. (dir.), Les marges de l'Armorique à l'âge du Fer. Archéologie et Histoire; culture matérielle et sources écrites (actes du XXIII ${ }^{\mathrm{e}}$ colloque de l'AFEAF, Nantes 1999)], p. 103-117.

Leroux, G., I993 - Rennes, Vieuxville-Beaurade. Occupation humaine en Basse-Vilaine de la préhistoire à la fin du Moyen-Age, Fouilles de sauvetage sur l'emplacement de la station d'épuration de l'agglomération rennaise, DFS de sauvetage urgent, Rennes, SRA Bretagne, AFAN, 70 p.

Leroux G., 2010 - Bretagne, Ille-et-Vilaine (Le Pertre, Brielles, Gennes-sur-Seiche, Argentré-du-Plessis). Terroirs gaulois, antiques et médiévaux sur le tracé de la LGV Rennes-Le Mans (Secteur 4), RFO - Diagnostic archéologique, Rennes, SRA Bretagne/ Inrap Grand-Ouest, 121 p.

Leroux G., Gautier M., Meuret J.-C. et NaAs P., i 999 - Enclos Gaulois et gallo-romains en Armorique, de la prospection aérienne à la fouille entre Blavet et mayenne, Rennes, éd. RAO, coll. "Documents archéologiques de l'Ouest", 335 p.

Maguer P. et Robert G., 2013 - "La maison gauloise dans l'ouest et le centre de la France ", in Krausz S., Colin A., Gruel K., Ralston I. et Dechezleprêtre T. (éd.), L'âge du Fer en Europe: Mélanges offerts à O. Buchsenschutz, Mémoires, Bordeaux, Ausonius Éditions, p. 247-258.

Menez Y., 2008 - Le camp de Saint-Symphorien à Paule (Côtesd'Armor) et les résidences de l'aristocratie du second âge du Fer en France septentrionale (thèse de doctorat), Paris, université de Paris 1 (Panthéon-Sorbonne), 2 vol.
Menez Y. et Lorho T., 2013 - «La Bretagne ", in Malrain F., Blancquaert G. et Lorho T., - " L'habitat rural du second âge du Fer: rythmes de création et d'abandon au nord de la Loire ", Recherches archéologiques 7, Paris, Inrap/CNRS, p. 170-191.

Mentele S., 20 I I - Bais, Carrière des Vallons, Un espace d'activité artisanale du second âge du Fer et de la période gallo-romaine précoce : une probable aire de traitement des denrées agricoles, RFO - Fouille archéologique, Rennes, SRA Bretagne/Inrap Grand-Ouest, 319 p.

Meuret J.-C., I993 - Peuplement, pouvoir et paysage sur la marche Anjou-Bretagne (des origines au Moyen Âge), Société d'Archéologie et d'histoire de la Mayenne, suppl. 4, 656 p.

Michel, M., 2013 - Torcé (35), La Grande Maçonnais 2, RFO Fouille archéologique, Rennes, SRA Bretagne/Éveha, 3 vol.

Pailler J.-M., 2006 - "Quand l'argent était d'or. Paroles de Gaulois ", Gallia, 63, p. 211-241.

Pouille D., 20I 3 - Brielles; La Massuère (35). Habitat et II âge du Fer et occupation médiévale et moderne, RFO - Fouille archéologique, Rennes, SRA Bretagne/Inrap Grand-Ouest, 250 p.

SicARD S., 2010 - Saint-Sauveur des Landes, "Les vairies, Le Croisé ". Ille-et-Vilaine Une occupation gauloise en milieu humide, RFO - Diagnostic archéologique, Rennes, SRA Bretagne, Inrap Grand-Ouest, 205 p.

TANGUY D., 2000 - « Le site d'habitat de l'âge du Fer de Kerven Teignouse à Inguiniel (Morbihan) ", Revue Archéologique de l'Ouest, 17, p. 143-173.

Toron S., 20 I 2 - Argentré-du-Plessis (35), La Blancharderie, RFO - Fouille archéologique, Rennes, SRA Bretagne/Éveha, 3 vol., 337 p. et $76 \mathrm{pl}$.

Toron S., 2014 - Domagné (35), La Lande Barbot, un établissement rural gaulois sur le tracé de la LGV Le Mans-Rennes, (RFO - Fouille archéologique), Rennes, SRA Bretagne/Éveha, 373 p.

VACHer S. et Bernard V., 2003 - Un site en zone inondable : Le grand Aunay à Yvré-l'Évêque, Revue archéologique de l'Ouest, suppl. 10 [Mandy B et de Saulce A. (dir.), Les marges de l'Armorique à l'âge du Fer. Archéologie et Histoire; culture matérielle et sources écrites (actes du XXIII ${ }^{\mathrm{e}}$ colloque de l'AFEAF)], p. 289-212. 
Zusammenfassung: La Blancharderie bei Argentré-du-Plessis (Ille-et-Vilaine, Frankreich): ein späteisenzeitlicher Fundplatz in einem Tälchen im Bereich der Schnellzugtrasse zwischen Rennes und Le Mans - Im Rahmen der Erschließungsarbeiten für die Schnellzugtrasse (LGV, Ligne à grande vitesse) zwischen Rennes und Le Mans wurde bei Argentré-du-Plessis (Ille-et-Vilaine, Bretagne) eine archäologische Ausgrabung durchgeführt. Unter den archäologischen Fundplätzen, die im Rahmen der verschiedenen Grabungen auf der Schnellzugtrasse untersucht werden konnten, zeichnet sich der Fundplatz von La Blancharderie durch die Besonderheit aus, das er sich im Bereich eines Talbodens in unmittelbarer Nachbarschaft des Zusammenflusses von zwei noch heute existierenden Bächen befindet. Im Randbereich dieses Tälchens, in unmittelbarer Nachbarschaft eines Feuchtgebietes, das im Laufe der Zeit mehrere Veränderungen erfahren hat, konnte die Ausdehnung einer eisenzeitlichen Siedlung festgestellt werden. Diese Siedlung war durchgehend vom Anfang des 3. Jh. v. Chr. bis zum Anfang des 1. Jh. v. Chr. besiedelt. Sie wird durch ein System gradlinig verlaufender Gräben gekennzeichnet, die eine große trapezförmige Einhegung begrenzten. Eine Seite dieser Einhegung konnte im Bereich der Grabung auf rund $850 m^{2}$ Grundfläche freigelegt werden, jedoch setzt sich die Anlage zweifellos in Richtung des südöstlich davon gelegenen Plateaus fort. Das wissenschaftliche Interesse an dieser Einhegung, neben ihrer Lage am Rande eines Feuchtgebietes, besteht in der Vielzahl der dort nachgewiesenen Baubefunde, die eine zeitliche und räumliche Abfolge von verschiedenen Siedlungseinheiten auf begrenztem Platz dokumentieren. Bei den Ausgrabungen konnten verschiedene Bauten mit Fundamentgräbchen und einer Wandkonstruktion aus aneinandergesetzten Wandpfosten sowie Pfostenbauten freigelegt werden.

Resumen: El Blancharderie en Argentré-du-Plessis (35): un sitio de fondo de valle al final de la Edad del Hierro en el trazado del tren de alta velocidad Rennes-Le Mans - La excavación arqueológica de Argentré-du-Plessis fue contratada como parte de la construcción de la linea del tren de alta velocidad Rennes-Le Mans. Entre los sitios descubiertos en esta trama lineal, él de la Blancharderie ofrece la particularidad de estar ubicado en el fondo del valle cerca de la confluencia de dos corrientes todavía activos. Bordeando la vaguada, en una zona húmeda que pasó por varias transformaciones, se ha puesto de manifiesto los límites de extensión de una institución gala que evoluciono continuamente por lo menos desde principios del siglo III a principios del siglo I antes de nuestra era. La ocupación se caracteriza por el establecimiento de un sistema de trincheras rectas que define un amplio recinto de forma trapezoidal. Su extremidad fue excavada en una superficie de $850 \mathrm{~m}^{2}$, mientras que sigue, sin duda alguna, la meseta al suroeste. El interés de este recinto, además de su ubicación en el borde de una zona húmeda, es la multiplicación de los edificios que revela la sucesión espacial y temporal de las casas en un pequeño espacio. Varias arquitecturas sobre trincheras de fundación, muros sobre postes y edificios sobre postes plantados se han despejado.

Schlüsselwörter: Bretagne, Feuchtgebiet, Talboden, Einhegung, Siedlung, Fundamentgräbchen, späte Eisenzeit, Keramik, Herdplatte, Mühlstein.

Palabras clave: Bretaña, zona húmeda, fondo de valle, recinto, hábitat, trinchera de fundacion, La Tène final, cerámica, placa de hogar, molino. 